\title{
Some properties of Cosmic String Junctions
}

\author{
Johanna Karouby
}

Master of Science

Physics

McGill University

Montreal,Quebec

2009-06-15

A thesis submitted to McGill University in partial fulfilment of the requirements of the degree of Master of Science

Copyright Johanna Karouby 2009 


\section{ACKNOWLEDGEMENTS}

I'd like to thank Alisha Wissanji, Loison Hoi, Paul Franche, Aaron Vincent and many other people of the 3rd floor for creating a good and agreable working atmosphere.

I am also grateful to Hassan Firouzjahi for our collaboration on different projects.

I finally wish to thank my supervisor, Robert Brandenberger, for his patience and the freedom he gives me in my research. 


\section{CONTRIBUTIONS OF AUTHORS}

In this thesis, chapter 2 and 3 are extracted from two articles in collaboration with Robert Brandenberger, Hassan Firouzjahi ${ }^{c}$ and Shahram Khosravid ${ }^{d, e}$ who contributed to the last article.

${ }^{c}$ School of Physics, Institute for Research in Fundamental Sciences (IPM), Tehran, Iran.

${ }^{d}$ School of Astronomy, IPM, Tehran, Iran.

${ }^{e}$ Physics Department, Faculty of Science, Tarbiat Mo'alem University, Tehran, Iran Hassan and I (in the second paper joined by Shahram) worked through the computations completely independently in order to double check everything. The whole supervision was done by Hassan Firouzjahi and by Robert Brandenberger. 


\begin{abstract}
Cosmic strings are linear concentrations of energy of macroscopic size. Cosmic superstrings come from string theory and can form junctions. In the introduction, we define more precisely the strings, their formation and the main properties of single straight cosmic strings or superstrings. Then, we focus on those latter and study some major properties of junctions. As a first step, we find the metric close to a junction of strings in the weak-field approximation. We then find some signatures that would reveal their presence : Namely, the lensing that they create and the shift in photon wavelength passing through (leading to the Kaiser-Stebbins effect) in chapter 2, and the gravitational radiation they can emit in chapter 3 .
\end{abstract}




\begin{abstract}
ABRÉGÉ
Les supercordes cosmiques correspondent à des concentrations linéaires d'énergie emprisonnée de taille macroscopique. Les supercordes cosmiques, sont celles resultant de la théorie des cordes et peuvent former des jonctions. L'introduction definit plus précisément les cordes, leur formation et les principales propriétés des cordes ou des supercordes droites. Ensuite, on se focalise sur ces dernières en étudiant les principales propriétés des jonctions. Un premier pas consiste à trouver la métrique proche d'une jonction de cordes dans l'approximation de champ faible. Ensuite on trouvera des possibles signatures qui révèleraient leur présence : L'effet de lentilles qu'elles créent et le décalage dans la longueur d'onde des photons passant aux alentours (ce qui mène à l'effet Kaiser-Stebbins) au chapitre 2, et la radiation gravitationelle qu'elles peuvent émettre au chapitre 3.
\end{abstract}


TABLE OF CONTENTS

ACKNOWLEDGEMENTS .................... ii

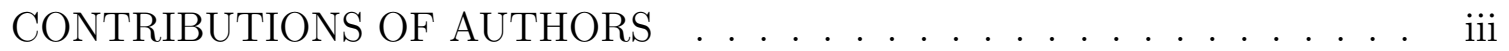

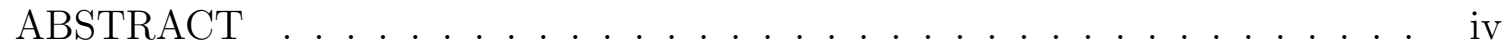

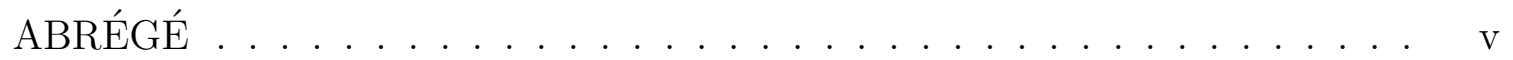

LIST OF FIGURES . . . . . . . . . . . . . . . . . . . . viii

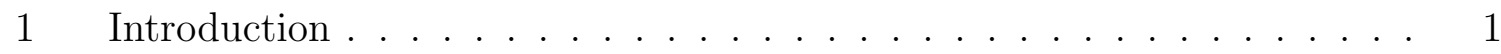

$1.1 \quad$ Introduction . . . . . . . . . . . . . . . . . . 1

1.2 Cosmic strings . . . . . . . . . . . . . . . . . . . 2

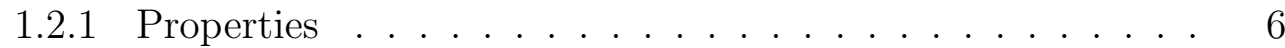

1.2 .2 Observational signatures . . . . . . . . . . . . . 8

1.2 .3 The abelian Higgs model . . . . . . . . . . . . . . 8

1.3 Cosmic superstrings . . . . . . . . . . . . . . . . . . . . . . 9

1.4 Strings formation . . . . . . . . . . . . . . . . 11

1.4.1 Phase transitions . . . . . . . . . . . . . . . . 11

1.4.2 The Kibble mechanism . . . . . . . . . . . . . . . . . 15

1.5 Formation of cosmic superstrings . . . . . . . . . . . . 16

1.6 Scaling solution . . . . . . . . . . . . . . . . . 17

1.7 Lensing due to the presence of an abelian straight cosmic strings . 19

1.8 String Doppler shifts : The Kaiser and Stebbins effect . . . . . . . 23

1.9 Gravitational radiation emitted by excited abelian cosmic strings . 25

2 Lensing and CMB Anisotropies by Cosmic Strings at a Junction . . . . . 27

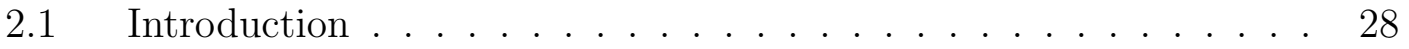

2.2 The $\operatorname{setup} \ldots \ldots \ldots \ldots \ldots \ldots$

2.3 The flatness of the geometry . . . . . . . . . . . . . 33

2.4 The Propagation of Light . . . . . . . . . . . . . . . . . 34

2.5 CMB anisotropies and cosmic String wakes . . . . . . . . . 41 
2.6 Conclusions . . . . . . . . . . . . . . . . . 45

2.7 Acknowledgments . . . . . . . . . . . . . . . . . 46

REFERENCES . . . . . . . . . . . . . . . . . . . 47

3 Gravitational Radiation by Cosmic Strings in a Junction . . . . . . . . . 51

3.1 Introduction . . . . . . . . . . . . . . . . . . . . . 52

3.2 The $\operatorname{Setup} \ldots \ldots \ldots \ldots \ldots$. . . . . . . . . . . . . 54

3.3 Examples ......................... 59

3.3.1 A semi-infinite string attached to the wall . . . . . . . . 59

3.3.2 Strings at a junction . . . . . . . . . . . . . 63

3.4 Discussion . . . . . . . . . . . . . . . . . . . . 66

3.5 Acknowledgments . . . . . . . . . . . . . . . . . 69

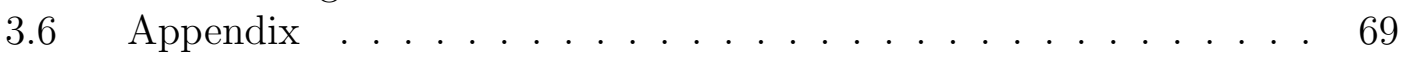

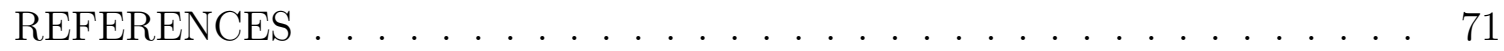

4 Conclusion and prospects . . . . . . . . . . . . . . . . 74

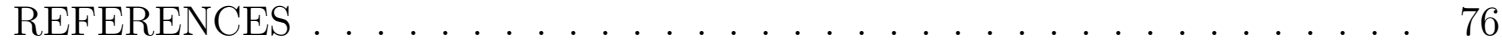


1-1 Point defects in a liquid crystal $[19,20] \ldots \ldots$

1-2 The Mexican hat potential . . . . . . . . . . . . . . . 5

1-3 When cosmic strings collide they can exchange partner a) or they can self-intersect b.1) and form loop b) . . . . . . . . . . 7

1-4 Formation of a FD-string (or(1,1)-string) leading to a 3-string junction 11

1-5 The Coleman - Weinberg potential energy of the Higgs field $\phi$ at various temperatures . . . . . . . . . . . . . . . . . 12

1-6 The potential energy of the Higgs field $\phi$ for a second order phase transition at temperatures near $T c \ldots . . . . . . . .14$

1-7 The Kibble mechanism for cosmic strings. $\xi$ is the correlation length $[25] \ldots \ldots \ldots \ldots \ldots$

1-8 Scale-invariant evolution of a cosmic string network [25] . . . . . . . 18

1-9 The metric around a cosmic string is conical, meaning that there is a deficit angle : when we go around the string, we need less than $2 \pi$ to come back at the same initial position[26]. . . . . . . . . . . 20

1-10 The Kaiser-Stebbins effect is originated from conic space-time around the cosmic string. A moving cosmic string induces relative speed between the light source and observer and causes a Doppler shift of photons. In the figure, $\Delta$ represents the effective deficit angle viewed from observer[26]. . . . . . . . . . . . . . 
2-1 In this figure two parallel light rays emitted from infinity are deflected towards the point $\mathrm{G}$. The contour $\mathrm{C}$ is made of the point $\mathrm{G}$, the light rays and the line connecting the two light rays distance at infinity, denoted by the dashed line. The strings cross the plane spanned by the contour at the points indicated by the cross signs. Only those strings which are enclosed by the contour contribute in (2.19) and (2.25). . . . . . . . . . . . . . . . 35

2-2 In this figures the multiple lensings by $N$ co-planar strings at a junction is sketched. The object $\mathrm{A}$ and its lensing counterparts $A_{i}, i=1 . . N-1$, form a closed loop. Each image is the source for the nearby images via the enclosed string. For example, in the three-string junction in the left figure, the object $\mathrm{A}$ is the source for the image $A_{1}$ via the string $\mu_{1}, A_{1}$ is the source for the image $A_{2}$ via the string $\mu_{2}$ and $A_{2}$ is the source for the object A via the string $\mu_{3} \ldots \ldots \ldots \ldots \ldots \ldots \ldots \ldots \ldots \ldots$ 


\section{CHAPTER 1 Introduction}

\subsection{Introduction}

Cosmic strings have been studied for a long time. In the 1980's, it was thought that they could provide a mechanism of structure formation as an alternative to inflation [1]. But in the late 1990's, observations of the cosmic microwave background (CMB) anisotropies by the Boomerang experiment [2] revealed a sharp acoustic peak in the angular power spectrum which is not predicted by cosmic string models and thus proved that cosmic strings could only have a minor contribution to structure formation (less than 10\% [3]).

There has been a renewal of interest in cosmic strings beginning around 2000, when it was shown that cosmic strings are often produced at the end of inflation in supersymmetric grand unified models [4]. An additional impetus to consider cosmic strings came after the realization that string theory contains non-perturbative objects called D-branes, and when string theorists started thinking about brane inflation models [5] in which the collision of branes at the end of inflation automatically lead to creation of cosmic D-superstrings [6]. As pointed out much earlier [7] there is another

kind of cosmic string which comes directly from string theory, namely cosmic Fsuperstrings (fundamental superstrings of macroscopic size). It was recently realized that such cosmic superstrings can be stable on cosmological scales [8]. Hence, there is renewed interest in searching for observational signatures of cosmic strings. 
There are well-known specific observational signatures of cosmic strings (lensing, Kaiser and Stebbins effect, gravitational radiation...). We are interested in signatures which are specific to cosmic superstrings and distinct from those arising from cosmic strings in simple field theory models. The detection of such signatures would provide strong observational support for string theory as the fundamental theory of Nature.

Studying string junctions is a good way to reach this goal. Since such junctions arise in superstring theory, but cannot form in simple field theory models yielding cosmic strings, finding signatures of them would allow a possible detection in the case where they exist.

We start, in Chapter 2, by finding the metric of a junction. This is the main tool to derive other useful means of detecting string junctions like those mentioned above. Then we will go in more details and study the propagation of light (lensing) and the CMB anisotropies in the presence of a string junction in order to see how string will modify the usual predictions of cosmic strings (Kaiser and Stebbins effect [9]). The third chapter is dedicated to the study of the emission of gravitational radiation by a semi-string attached to a wall and its generalization by string junctions.

\subsection{Cosmic strings}

Cosmic strings can be seen as linear concentrations of trapped energy. An analogy with condensed matter physics can be used to understand them: they are like the disclination lines in some liquid crystals where the molecules do not have the

same orientation. Cosmic strings are one dimensional topological defects [13] that form during phase transitions as the universe cools down. In quantum field theory, there are different types of topological defects of different dimensions : In three 
spatial dimensions, the stable defects can be point-like (monopoles), linear (strings) or planar (domain walls). Which kind of defects form in a particular field theory model depends on which symmetry is broken[13, 17, 18].

Figure 1-1 is a picture of a liquid crystal that has been cooled rapidly and underwent a phase transition. This phase transition generated topological defect points, located at points in the image where four dark regions converge. The original picture was colored and showed the different polarization of light corresponding to the alignment of particles in the liquid crystal. As you "orbit" one of the topological defects, the orientation of the molecules goes through one complete 360-degree rotation.

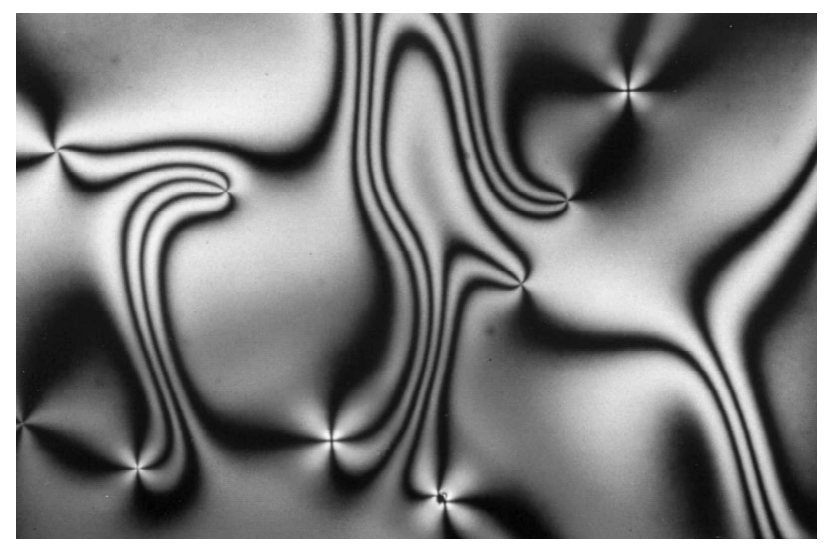

Figure 1-1: Point defects in a liquid crystal [19, 20]

A simple model is the $\mathrm{U}(1)$ abelian string when the general group which gets broken is $\mathrm{U}(1)$. To understand it, we can consider a model in which the symmetry breaking is realized by means of a Higgs scalar field doublet with a "Mexican hat" potential. For example, we can choose the Goldstone model of a scalar field $\phi$. 
Our Lagrangian density is :

$$
L=\partial_{\mu} \bar{\phi} \partial^{\mu} \phi-V(\phi)
$$

where $V(\phi)$ represents the Mexican hat potential :

$$
V(\phi)=\frac{1}{4} \lambda(\bar{\phi} \phi-\eta)^{2}
$$

where $\lambda$ is a dimensionless coupling constant and $\eta$ denotes the symmetry breaking amplitude of the field $\phi$.

The space of ground states (vacuum manifold) is characterised by a field value which minimizes the potential $V(\phi)$. In our model, this happens when the field value takes on its vev (vacuum expectation value). This occurs for a certain value of $\phi$ different from 0 , namely $\phi=\phi_{0}$ with $\left|\phi_{0}\right|=\eta$. [13, 15]. This model is invariant under the group $\mathrm{U}(1)$ of global ( $\alpha$ is a constant in space) phase rotations :

$$
\phi \rightarrow e^{i \alpha} \phi
$$

The ground state will correspond to one random point on the circle of minima ( of radius $\eta$ ):

$$
<0|\phi| 0>=\eta e^{i \theta}
$$

where $\theta$ is a certain angle.

This state is not invariant under the transformation (1.3): we say that the symmetry is spontaneously broken. Introducing two real fields $\phi_{1}$ and $\phi_{2}$ via $\phi=\phi_{1}+i \phi_{2}$, let us for the moment assume that the ground state is taken on at $\phi_{1}=\eta$ and $\phi_{2}=0$. Now, $\phi_{1}$ fluctuations correspond to oscillations around this point and $\phi_{2}$ 
to the motion around the circle of minima of the potential. In this case, one can show [13] that $\phi_{1}$ excitations represent a massive particle whereas $\phi_{2}$ excitations are massless.

We can plot the potential in 3 dimensions :

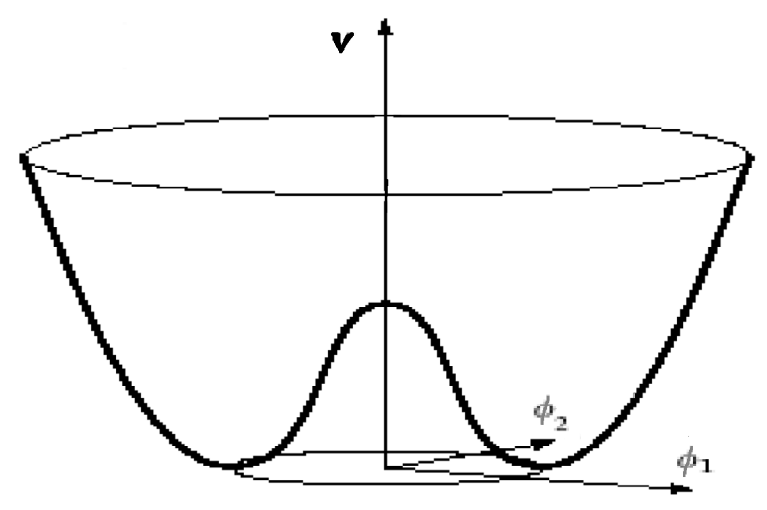

Figure 1-2: The Mexican hat potential

The points at the minimum of the potential are equivalent. There is thus no reason one should be preferred over the other. A cosmic string configuration is obtained when the field value rotates about the minimum of the potential as we move around a circle $\mathcal{C}$ in space. If this is the case, then field continuity implies that there must be a point in space on the disk bounded by the circle $\mathcal{C}$ where the field vanishes. This is a point on the defect. At this point there is potential energy. It is trapped because the zero of the field cannot be removed. We can deform the disk in perpendicular direction keeping the boundary $\mathcal{C}$ fixed, and the same continuity argument as before implies that there : defect points line up into a string. A cosmic string configuration is characterized by the vanishing of the field $\phi$ in the core of the string. The string has a microscopic width which is given by $\eta^{-1}$. 
At high temperature, the fluctuations in $\phi$ are big and there is no problem for $\phi$ to go over the central hump. There is no preferred direction so there is a rotational symmetry. At temperatures lower than a critical value, the field won't be able to go over the hump anymore and will relax to one of its possible ground states on the circle of minima. Thus, the symmetry is broken [15].

At points separated by more than a certain correlation length (which by causality is always smaller or equal to the horizon) the field $\phi$ will choose to be at angles $\theta$ in the vacuum manifold. These are uncorrelated since different pieces far from each other don't talk to each other. Thus, there is a finite probability that about a circle in space with a radius greater than the correlation length the field $\phi$ will wind once (or more times) about the vacuum manifold. This implies that a defect string has formed. This is the Kibble mechanism of defect formation ([14]) which will be described in more detail later on in the Introduction.

When the system cools down, there will be a tendency for the angle to become more uniform since the energy of the system depends on the gradient of $\phi$. Still, irregularities will remain and there will be different $\theta$ for different point in space : Linear defects may survive until the present time.

\subsubsection{Properties}

We will assume no breaking of strings to ensure their stability on cosmological time scale. Strings from gauge theory cannot break whereas in case of cosmic superstrings, the stability of the strings is a non-trivial assumption [8].

The key property of cosmic strings and superstrings which we assume is realized

is the "scaling solution", the statement that at all cosmological times there will be 
a network of strings whose correlation length is comparable to the horizon. This scaling solution is maintained by collisions (inter-commutation) of cosmic strings. When two long strings intersect, the strings will exchange their segments, with a probability equal to around 1.

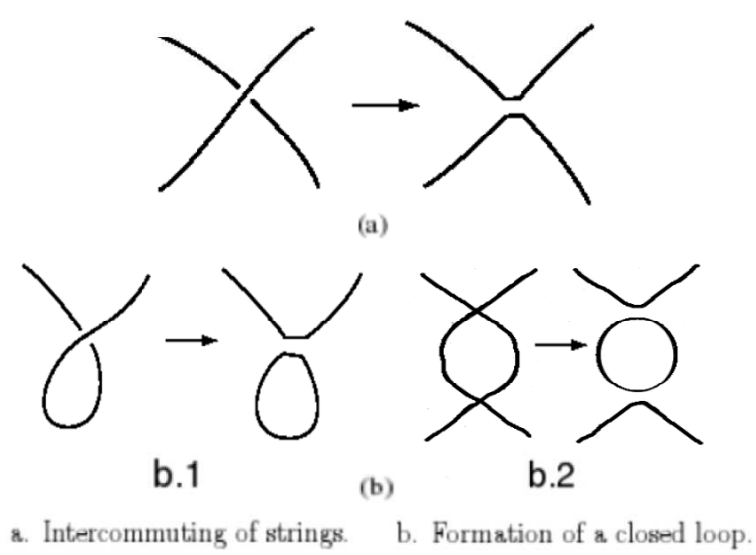

Figure 1-3: When cosmic strings collide they can exchange partner a) or they can self-intersect b.1) and form loop b)

In this way, a cosmic string loop can be formed (see figure 1-3). Loop production yields the energy loss mechanism which allows the scaling solution to be maintained. Loops of strings that will oscillate, emit gravitational radiation and shrink. There can also be kinks formed on each strings that will radiate gravitational waves. Strings can also give rise to particle production. One model proposes that when strings overlap near cusps, the cusp region can annihilate emitting particles [13].

Summarizing, in an expanding universe, the long string network will expand on a scale comparable with the horizon scale $t$. Inside the horizon, the strings will be straighten [12]. This is why we study straight cosmic strings. 


\subsubsection{Observational signatures}

In the following, we will see that there are several ways of detecting long almost straight cosmic strings. We will explain in more detail the Doppler redshift of light via the so-called KS effect $(1.8,2.5)$, and the specific lensing signatures $(1.7,2.4)$. They are both due to the deformation of space created by such a string (figure 1-9). We will also study gravitational radiation $(1.9,3)$ emitted by wiggly strings. These features are present both for cosmic superstrings and for regular field theory cosmic strings. This thesis will focus on the role of string junctions predicted by string theory but not present in simple field theory models of cosmic strings.

\subsubsection{The abelian Higgs model}

We have to distinguish between local (or gauge) and global strings. Previously, we have explained the Goldstone model which is a global model. Since local gauge theory play a central role in particle physics, we now study a simple example of this sort : the abelian Higgs model. The Lagrangian density is :

$$
L=\bar{D}_{\mu} \bar{\phi} D^{\mu} \phi-V(\phi)-\frac{1}{4} F_{\mu \nu} F^{\mu \nu}
$$

where $D_{\mu}=\partial_{\mu}-i e A_{\mu}$ and $F_{\mu \nu}=\partial_{\mu} A_{\nu}-\partial_{\nu} A_{\mu}$.

$A_{\mu}$ is the gauge vector field and $e$, the gauge coupling.

This model is invariant under the group $\mathrm{U}(1)$ of local $(\alpha(x)$ depends on the space-time position) phase transformations :

$$
\phi(x) \rightarrow e^{i \alpha(x)} \phi(x) \quad, \quad A_{\mu}(x) \rightarrow A_{\mu}(x)+\frac{1}{e} \partial_{\mu} \alpha(x)
$$


Taking the same Mexican hat potential as before, the symmetry is also spontaneously broken and the field $\phi$ acquires a vacuum expectation value. Rewriting the

Higgs field, $\phi=\eta+\frac{\phi_{1}}{\sqrt{2}}$, we get a new form for the Lagrangian where we can see that the gauge vector field, $A_{\mu}$, becomes massive.

Finally, there are also more complicated models with non-abelian cosmic strings. For example, we would have to use in the above Higgs model:

$D_{\mu}=\partial_{\mu}-i e A_{\mu}^{a} T^{a}$ and $F_{\mu \nu}=\partial_{\mu} A_{\nu}-\partial_{\nu} A_{\mu}+e f_{a b c}$

where $T^{a}$ are the generators of the representation of $\mathrm{G}$, the group of gauge transformation leaving our Lagrangian invariant (U(1) in the Goldstone and abelian Higgs model) and $f_{a b c}$ are the struture constants of G.

\subsection{Cosmic superstrings}

They are strings coming from superstring theory. Our assumption here, is that there are no NS-NS (Neveu Schwarz) or Ramond-Ramond sources in the background. We can therefore describe them by simply using Nambu-Goto action :

$$
S=-\mu \int d t d \sigma \sqrt{-|\gamma|}
$$

where $\mu$ is the tension of the string and $\gamma_{m n}$ is the metric induced on the string

$$
\gamma_{m n}=g_{\mu \nu} \partial_{m} X^{\mu} \partial_{n} X^{\nu}
$$

and $|\gamma|$ is its determinant. Here $m, n=\{t, \sigma\}$ are the coordinates along the string worldsheet, $X^{\mu}$ are the space-time coordinates and $g_{\mu \nu}$ is the space-time metric.

An important property of cosmic superstrings is that they can form junction, and in the following chapters we will study them in more details. 
There are two main kinds of strings coming from the brane inflation scenario (see section 1.5 for more details on their formation). Beside the fundamental strings of string theory or F-strings, there are also D1-branes or D-strings.

- The fundamental strings or F-strings

They are the fundamental objects in string theory. They are quantum mechanical objects that define a weakly coupled string theory

- The Dirichlet strings or D-strings

In some type of string theory like type IIB, the brane world idea leads to the possibility of having D-branes (where D stands for Dirichlet boundary condition) on which strings would be attached[15]. There are one dimensional D-branes called D1-branes or D-strings. Another way of getting D-strings is to wrap the extra dimensions of the D-branes in order to be left with linear objects in our regular $3 \mathrm{~d}$ space, the D-strings. These are more similar to the usual cosmic strings, are essentially classical and, like D-branes, have a conserved charge.

- The (p,q)-strings

D- and F- strings are different types of objects but can form bound state of p F-string and q D-string, called (p,q)-strings. The simplest case is the (1,1)string. This is so because F- and D- strings are different objects and cannot intercommute. Instead they will form a composite FD-string (also called (1,1)string) (see figure 1-4).

The following figure shows how a junction can form, leading to the creation of this new type of strings. 


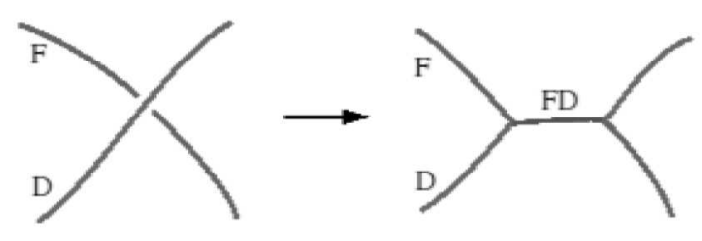

Figure 1-4: Formation of a FD-string (or(1,1)-string) leading to a 3-string junction

In the next two sections, we explain, physically and mathematically, how cosmic strings and cosmic superstrings could be created. The first ones can be better understood by a condensed matter analogy, whereas the latter require the use of a string theory scenario like brane inflation .

\subsection{Strings formation}

Phase transitions are essential to understand the creation of cosmic strings. Another point of view uses the Kibble mechanism that involves the notion of Manifold and symmetry breaking to explain the formation of topological defects.

\subsubsection{Phase transitions}

When the universe cools down below the critical temperature Tc, the Higgs field $\phi$ develops an expectation value $<\phi>$ corresponding to the ground state : Some symmetries are broken and a topological defect appears[13, 17]. The particle masses are determined by this value and the free energy of the system $(F=E-T S)$ becomes a non-trivial function of $\phi$. If the Higgs field $\phi$ becomes smaller, the particle masses typically decrease, the available phase space is larger and the entropy grows. $<\phi>$ has a tendency to decreases when the temperature increases and vanishes completely for $T=T c$.

They are two types of phase transitions that we describe below. 


\subsubsection{1st order phase transitions}

First order phase transition are extremely inhomogeneous in space. The expectation value of the scalar field, $\langle\phi(x)>$, is a discontinuous function fo time. In this process, quantum mechanics is essential.

The first-order phase transitions are those that involve a latent heat. During such a transition, a system either absorbs or releases a fixed (and typically large) amount of energy. During this process, the temperature of the system will stay constant as heat is added.

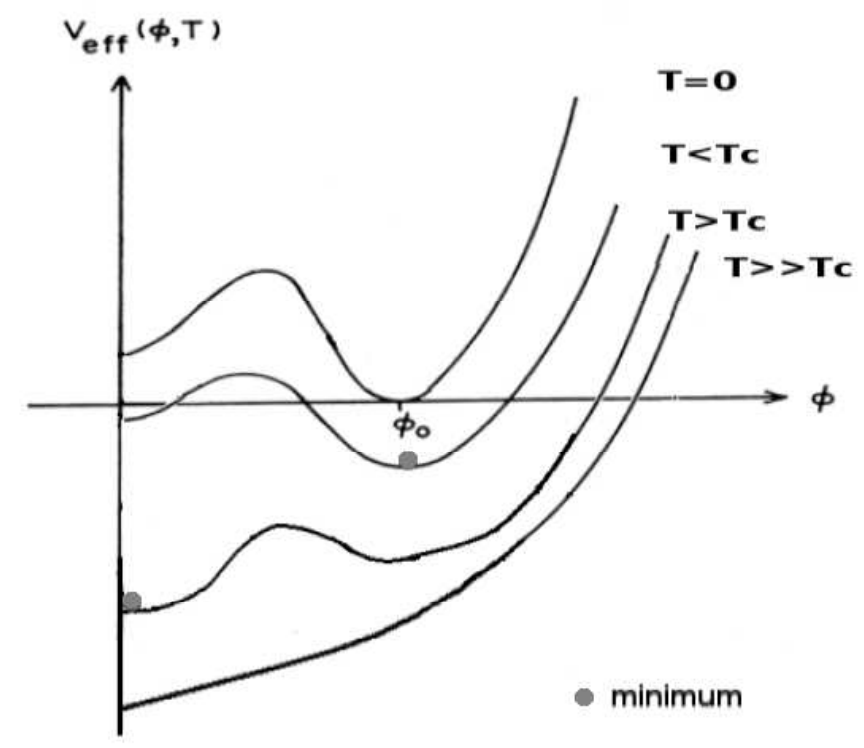

Figure 1-5: The Coleman - Weinberg potential energy of the Higgs field $\phi$ at various temperatures

The transition is discontinuous and we have to introduce radiative correction. The effective potential can therefore be expanded as :

$$
V e f f(\phi)=V(\phi)+V 1((\phi)+\ldots
$$


Where V1 is the one loop contribution to $\operatorname{Veff}(\phi)$ As an example, we can use the abelian Higgs model and consider the following potential : $V(\phi)=\mu^{2}|\phi|^{2}$. Depending on the value of $\mu$ and the renormalization scale we have different possibilities. For a certain range of values the minimum plunges deeper than the one at $\phi=0$, and the symmetry is broken. We choose to study a potential which possesses this feature at $\mathrm{T}=0$. In this case, we can write the effective potential as :

$$
V e f f=m^{2}(T)|\phi|^{2}+c|\phi|^{4} \ln \left(\frac{|\phi|^{2}}{\sigma^{2}}\right)
$$

where $\sigma$ is the renormalization scale, $m^{2}(T)=\mu_{0}^{2}+\frac{1}{4} e^{2} T^{2}$ is the mass square and c a constant. For $T>>T c$, Veff has a single minimum at $\phi=0$. Below $T c$, the minimum plunges below the symmetric minimum at $\phi=0$. One of the feature of 1 st order phase transition is that below $T c$, the ground state at $\langle\phi\rangle=0$ Is metastable. In this case it is also called "false vacuum". The false vacuum can decay to a lower energy state and cross the potential barrier by thermal fluctuation in the case where the supercooling of the universe is not too intense. If the transition occurs rapidly, then the false vacuum decay through the potential barrier using quantum tunneling .It gives rise to bubbles of the new phases. The transition is over when all the bubbles have collapsed,coalesced, and have reheated the matter.

\subsubsection{2nd order phase transitions}

Second order phase transitions are the continuous phase transitions. The transition occurs homogeneously in space (apart from the phase boundaries which become topological defects), and $\langle\phi(x)>$ evolves continuously in time. 
For a better understanding, we can consider the goldstone model (see section.1..) with Mexican hat potential. The high temperature effective potential in this model is :

$$
V e f f(\phi)=m^{2}(T)|\phi|^{2}+\lambda|\phi|^{4}
$$

where $m(T)^{2}=\frac{\lambda}{12}\left(T^{2}-6 \eta^{2}\right)$ is the mass square and $\eta$ the characteristic energy scale of symmetry breaking.

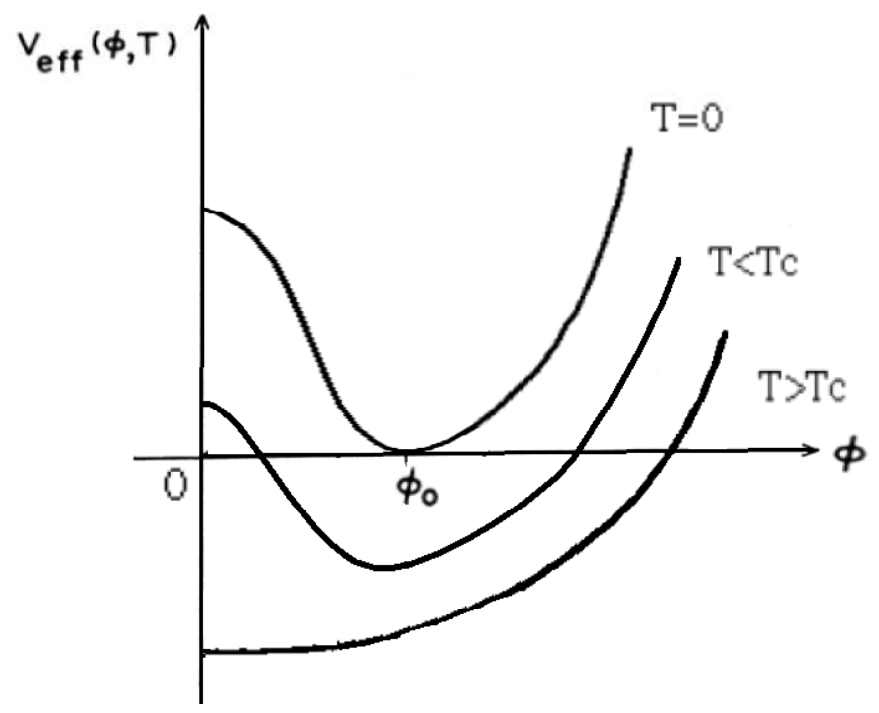

Figure 1-6: The potential energy of the Higgs field $\phi$ for a second order phase transition at temperatures near $T c$

For $T>T c$, the symmetry is restored since there is one unique minimum at $\phi=0$ therefore the expectation value vanishes. For $T<T c$, the minimum is no more one point but there is a whole circle of minima : the potential will choose one of them : we get a vev (vacuum expectation value). Uncorrelated region, will get different vev and will wind around one dimensional defect :therefore strings can form (see figure 1-7). Defect network can survive below $T c$, only because the field $\phi$ 
doesn?t have enough time to equilibrate on scales greater that the correlation length $\xi(t)$. Thermal fluctuation can take $\phi$ over the potential barrier and can unwind the topological defect. For a second order phase transition the stability is obtained for the Ginzburg temperature : $T=T_{G}$.

In the following section, we study in more details how the symmetry of the potential can be broken, yielding to creation of topological defects.

\subsubsection{The Kibble mechanism}

The kibble mechanism states that the existence of defects structures depends on the topology of the vacuum manifold, $\mathcal{M}$, the space of minimum energy density configurations (ground state). Cosmic strings arise from the symmetry breaking from a group G (we can consider $\mathrm{U}(1)$ ) down to a subgroup H. They are one dimensional topological defects formed in the case where $\mathcal{M}=\mathrm{G} / \mathrm{H}$ is such that the first homotopy group, $\Pi_{1}(\mathcal{M})$, is non trivial : $\Pi_{1}(\mathcal{M}) \neq I$. $[12,17]$. This is equivalent to say that $\mathcal{M}$ is not simply connected (it contains unshrinkable loops).

The Mexican hat potential is a good example of $\mathrm{U}(1)$ symmetry breaking. In this case, the vacuum manifold is a circle : $\mathcal{M}=S^{1}$ since the minima of energy lies on a circle (see 1.2).

This simple picture shows that around a string there is a non-trivial winding: There are uncorrelated regions with different phases winding around the strings. We

can define the correlation length, $\xi$, as the length scale above which the phase of the scalar field $\phi$ are uncorrelated. 


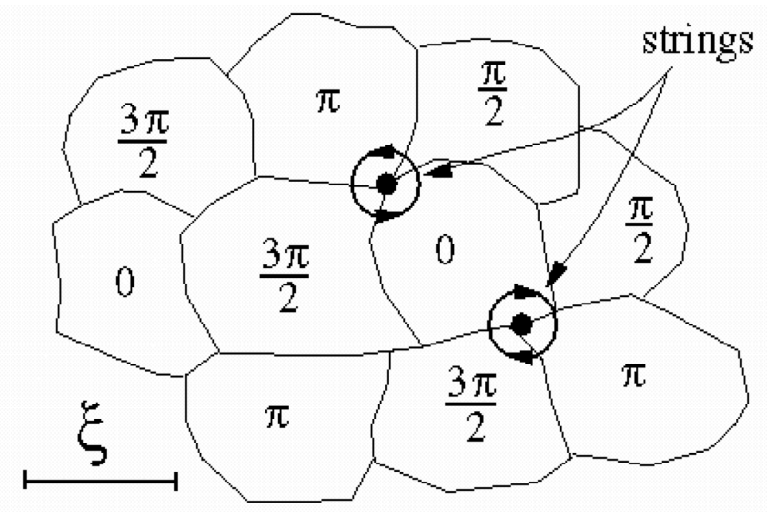

Figure 1-7: The Kibble mechanism for cosmic strings. $\xi$ is the correlation length $[25]$

Whenever a $\mathrm{U}(1)$ symmetry becomes broken during the evolution of the universe, a network of strings must actually form with a characteristic lenght scale of order $\xi[13]$. Since correlations cannot establish with a speed greater that the speed of light, $\xi(t)$ cannot exceed the causal horizon, $d_{H}(t) \sim t$.

The Kibble mechanism is not only valid for cosmic strings but also for cosmic superstrings. In the following section, we explain more clearly how F-and D-strings form in the context of the brane world scenario.

\subsection{Formation of cosmic superstrings}

In the brane inflation scenario, strings can also arise from a symmetry breaking that leave an unbroken $\mathrm{U}(1)$ symmetry.

If branes are D-branes, annihilation of one brane and one anti-brane creates two different types of strings. On each of the branes there is a $\mathrm{U}(1)$ gauge symmetry, so the total gauge group is $\mathrm{U}(1) \mathrm{x} \mathrm{U}(1)$. One linear combination of the $\mathrm{U}(1), A=$ 
$A 1-A 2$, is broken when the tachyon condenses and will lead to the creation of a network of D-strings according to the Kibble argument. The other one, orthogonal, $\tilde{A}=A 1+A 2$, is not higgsed but confined (non dynamical) . So the wave function of $\tilde{A}$ vanishes when the tachyon vanishes. After the branes annihilate, this field must be squeezed into electric flux tube : those are the F-strings ([11],[21]).

In cosmology, D-strings refer not only to D1-branes, but to $\mathrm{D}(1+\mathrm{k})$-branes that are wrapped on $\mathrm{k}$ small dimensions and therefore look one dimensional in three large dimensions. This comes from the fact that annihilation of D-branes produces defect of codimension 2 :

$$
D(3+k)+D(3+k) \rightarrow D(1+k)
$$

where $\mathrm{k}$ is the number of small dimension on which the branes are wrapped.The product $D(1+k)$ corresponds to cosmic D-strings.

This process produces only strings and not cosmological higher dimensional defects like monopoles or domain walls since only a one dimension of the $\mathrm{D}(1+\mathrm{k})$ brane is visible (the remaining $\mathrm{k}$ dimensions are compactified).

\subsection{Scaling solution}

Scaling solution is an important characteristic of strings network. It corresponds to an equilibrium where the network look the same at any time. There are two antagonist mechanisms that happen to reach it : loss of energy and growth of the typical length between the strings. The scaling solution is an attractor : if we start with too many strings, the higher collision rate will reduce the energy density. If we lack strings, there will be less likely to collide and the energy density will grow until it reaches the scaling solution. 
More precisely, the production of strings will increase the density of strings in the network. This will increase the probability for the strings to collide and therefore more loops will be created via loop production (see 1-3 ,b). These loops will oscillate and lose energy by gravitational radiation. Finally, it is possible to reach a kind of equilibrium where the ratio of the energy density in long strings, $\rho_{\text {inf }}(t)$, over the background energy density, $\rho_{c}(t)$, is constant in time : This is called the scaling solution.

Another approach is to use the correlation length of the network, $\xi(t)$, that can be seen as the typical distance between the strings. As we said before in section 1.4, correlations cannot establish with a speed greater that the speed of light and so $\xi(t)$ cannot exceed the causal horizon, $d_{H}(t) \sim t$. If it's the case, the string network is frozen, the network will expand as the scale factor, a(t), until the horizon catches up. If $\xi(t)$ is smaller than the horizon, strings will oscillate, the loop production and the number of collision will grow, and $\xi(t)$ catches up the horizon.

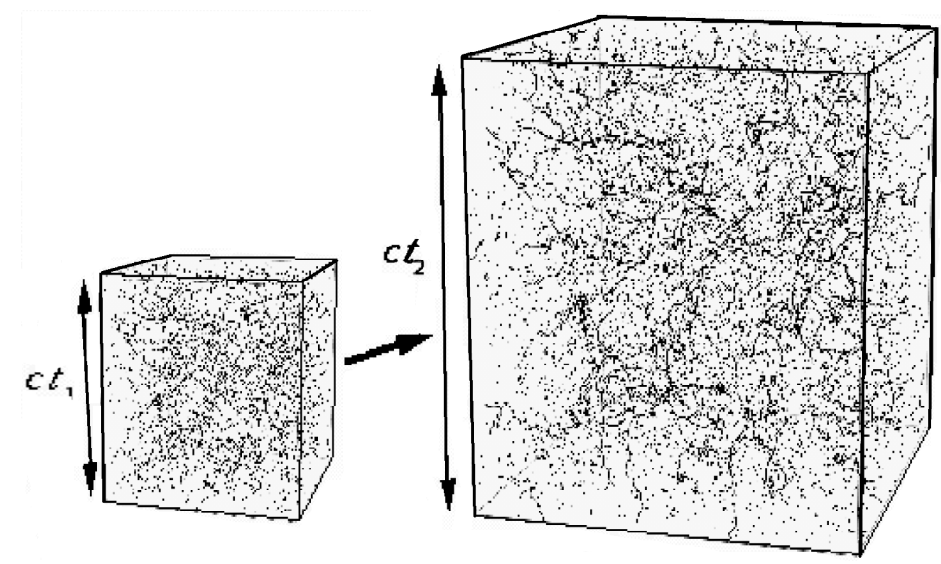

Figure 1-8: Scale-invariant evolution of a cosmic string network [25] 
For cosmic superstrings, the principle stays the same, except that you have to include the possibility of forming junctions. Numerical simulation show that in the horizon volume $t^{3}$, the total length of a string is proportional to t. So, the energy density for strings is $\rho_{s}=\frac{L \mu}{V} \propto \mu t^{-2}$. The scale factor is proportional to $t^{\frac{1}{2}}$ in the radiation dominated era and $t^{\frac{2}{3}}$ in the matter dominated era. Since the energy density scales like $a^{-4}$ for radiation and $a^{-3}$ for the matter, we get the energy density of the background, $\rho_{c}$, that is proportional to $t^{-2}$.

Therefore the ratio of $\frac{\rho_{s}}{\rho_{c}}$ is constant in both the radiation and the matter era. Simulations show that $\frac{\rho_{s}}{\rho_{c}}=60 G \mu$ during the matter dominated era and $\frac{\rho_{s}}{\rho_{c}}=400 G \mu$ during radiation dominated era, where $\mu$ is the string tension and $\mathrm{G}$ the universal gravitational constant.

In the last three sections, we study signatures of cosmic strings in the single string case, in order to generalise it later to the junction case in chapter 2 and 3 : Namely the lensing, the doppler shift of light inducing anisotropies in the temperature background (Kaiser-Stebbins effect) and finally, the gravitational emission.

\subsection{Lensing due to the presence of an abelian straight cosmic strings}

The presence of the string creates a conical space. That means that there is a deficit angle, $\Delta$, and going around a cosmic string doesn't require a 360 degrees rotation but only a $(2 \pi-\Delta)$ one. As a consequence,there is a lensing where we would see two exact identical images (possibly shifted with respect to each other).This would be a good signature to observe them. 


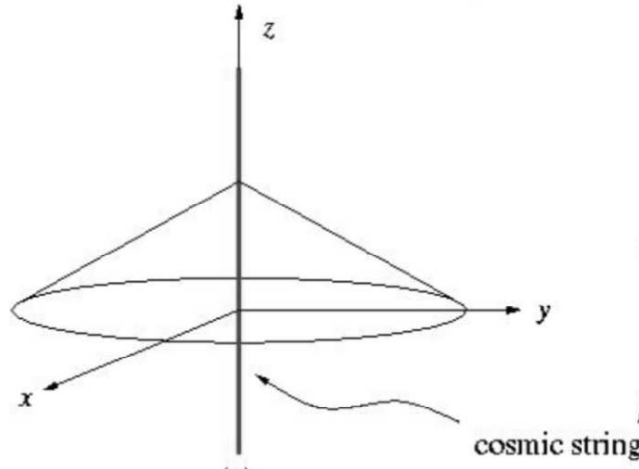

(a)

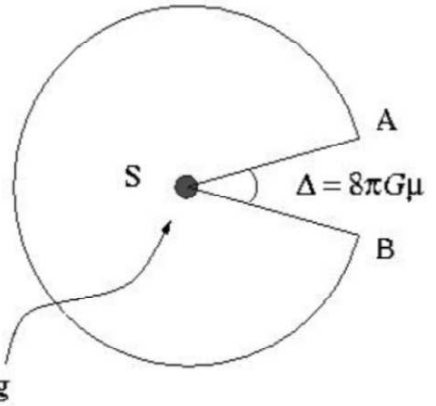

(b)

Figure 1-9: The metric around a cosmic string is conical, meaning that there is a deficit angle : when we go around the string, we need less than $2 \pi$ to come back at the same initial position[26].

In the following we compute the deficit angle for a static straight cosmic string. The first step is to derive the metric around a straight cosmic string:

Using the weak field approximation we have :

$$
g_{\mu \nu}=\eta_{\mu \nu}+h_{\mu \nu}
$$

where $h_{\mu \nu}$ is the perturbation due to cosmic strings, $\left|h_{\mu \nu}\right|<<1$ and $\eta$ is the flat background metric $\eta_{\mu \nu}=\operatorname{diag}(1,-1,-1,-1)$.

The linearized Einstein equations are :

$$
R_{\mu \nu}=8 \pi G\left(T_{\mu \nu}-\frac{1}{2} \eta_{\mu \nu} T\right) \equiv 8 \pi G S_{\mu \nu}
$$

where $R_{\mu \nu}$ is the Ricci tensor and $T=T_{\mu}^{\mu}$ is the trace of the energy-momentum tensor. 
The Einstein equations as usual are subject to the choice of gauge. We use the harmonic gauge $h_{\nu, \mu}^{\mu}-\frac{1}{2} h_{\mu, \nu}^{\mu}=0$ and then, Einstein equations (1.9) become:

$$
R_{\mu \nu}=-\frac{1}{2} \square h_{\mu \nu}
$$

where $\square$ is the four-dimensional Laplacian.

In the case of a string lying on the z-axis, the energy is localised and we can write the stress energy tensor as:

$$
T_{\mu}^{\nu}=\mu \delta(x) \delta(y) \operatorname{diag}(1,0,0,1)
$$

It can be shown (this computation is done in more details for a junction of strings in 2.2) that the solution of (1.9) and (1.10) is:

$$
\begin{aligned}
h_{t t} & =h_{z z}=0 \\
h_{x x} & =h_{y y}=8 G \mu \ln \left[\frac{\rho}{\rho_{0}}\right]
\end{aligned}
$$

where $\rho=x^{2}+y^{2}$ and $\rho_{0}$ is a constant of integration. Now that we have found the metric due to a straight and static cosmic string, we can write the line element :

$$
d s^{2}=d t^{2}-d z^{2}-(1-h)\left(d x^{2}+d y^{2}\right)
$$

where $h=8 G \mu \ln \frac{\rho}{\rho_{0}}$. In cylindrical coordinates this becomes:

$$
d s^{2}=d t^{2}-d z^{2}-(1-h)\left(d^{2} \rho+\rho^{2} d^{2} \phi\right)
$$


We are going to introduce new coordinates $\left\{\rho^{\prime}, \phi^{\prime}\right\}$ to rewrite the metric in a more meaningful way . If we take $d^{2} \rho^{\prime}=(1-h) d^{2} \rho$ and we integrate, we get :

$$
\rho^{\prime}=\int\left(1-\frac{h}{2}\right) d \rho=\rho-4 G \mu\left(\rho \ln \frac{\rho}{\rho_{0}}-\rho\right)
$$

In the case of a very low string tension we can make an approximation to the first order in $G \mu$ :

$$
\begin{aligned}
\rho^{\prime 2}(1-8 G \mu) & =\left(\rho^{2}-8 G \mu\left(\rho \ln \frac{\rho}{\rho_{0}}-\rho\right) \rho\right)(1-8 G \mu) \\
& \sim \rho^{2}-8 G \mu \ln \frac{\rho}{\rho_{0}} \rho^{2} \sim(1-h) \rho^{2}
\end{aligned}
$$

Taking $\rho^{\prime 2} d^{2} \phi^{\prime}=(1-h) \rho^{2} d^{2} \phi$ we obtain :

$$
(1-h) \rho^{2} d^{2} \phi=\rho^{\prime 2}(1-8 G \mu) d^{2} \phi=\rho^{\prime 2} d^{2} \phi^{\prime}
$$

That's why, we have : $d^{2} \phi^{\prime}=(1-8 G \mu) d^{2} \phi$ and so :

$$
\phi^{\prime}=(1-4 G \mu) \phi
$$

Our final metric in the new coordinates is : $d s^{2}=d t^{2}-d z^{2}-\left(d^{2} \rho^{\prime}+\rho^{\prime 2} d^{2} \phi^{\prime}\right)$.

We recognize the metric of a flat space but now the angle is constrained : When $\phi^{\prime}$ varies from 0 to $2 \pi, \phi^{\prime}$ varies from 0 to $2 \pi-8 \pi G \mu$. That's why there is a deficit angle equal to :

$$
\Delta=8 \pi G \mu
$$

We also study in more details this lensing in the junction case (section 2.1.1). Basically, the source will be lensed by the closest piece of string in the junction (to respect the weak field approximation the object must not be too far from the string) 
and the image obtained will be considered as the object for another part of the junction : there will be multiple images of the same object.

In addition to lensing, the space deformation due to the presence of a cosmic string implies some anisotropies in the temperature background : This is the Kaiser and Stebbins effect.

\subsection{String Doppler shifts : The Kaiser and Stebbins effect}

The Kaiser and Stebbins effect is due to the shift of photon passing through a cosmic string. There will be also a modification of the temperature map that will lead toa modified value of the CMB anisotropies.

To explain this phenomena we consider the example of two particles. The conical spacetime created by cosmic strings creates a shift in the velocity of particles moving toward the string. For simplicity, we consider two particles along parallel path orthogonal to the string and with the same velocity v. As they pass through the string they become closer to each other because of the deficit angle and will eventually collide. It can be shown that the relative velocity of the particles then becomes : $u=\gamma v \Delta$ where $\gamma=\left(1-v^{2}\right)^{-\frac{1}{2}}$ is the Lorentz factor and $\Delta$ is the deficit angle created by the cosmic string . Now, if we replace one particle by a light source and the other by an observer, the observer will see a discontinuous change in the frequency of light due to the Doppler effect. Physically, the observer will see an image

of the source and after the same image but with a shifted frequency. : $\frac{\delta \omega}{\omega}=\gamma v \Delta$. This also induces a discontinuous change of the background temperature across the 
string and we get for the temperature anisotropies:

$$
\frac{\delta T}{T}=\gamma v \Delta=8 \pi G \mu v \gamma
$$

This is the Kaiser-Stebbins effect. This can be generalized to cases where the velocities of the source and the observer, v (or equivalently when the velocity of the string in the reference frame where both the source and the observer don?t move) is not orthogonal to the string :

$$
\frac{\delta T}{T}=\gamma v \Delta=8 \pi G \mu v \gamma \mathbf{n} \cdot(\mathbf{v} \times \mathbf{s})
$$

Where $\mathbf{s}$ is a unit vector pointing along the string and $\mathbf{n}$ the one along the line of sight.

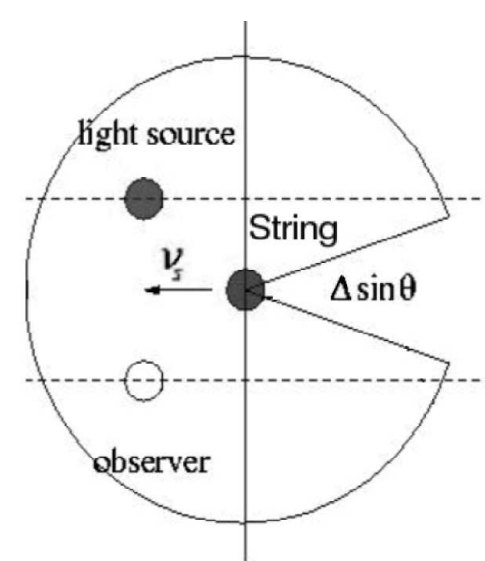

(a)

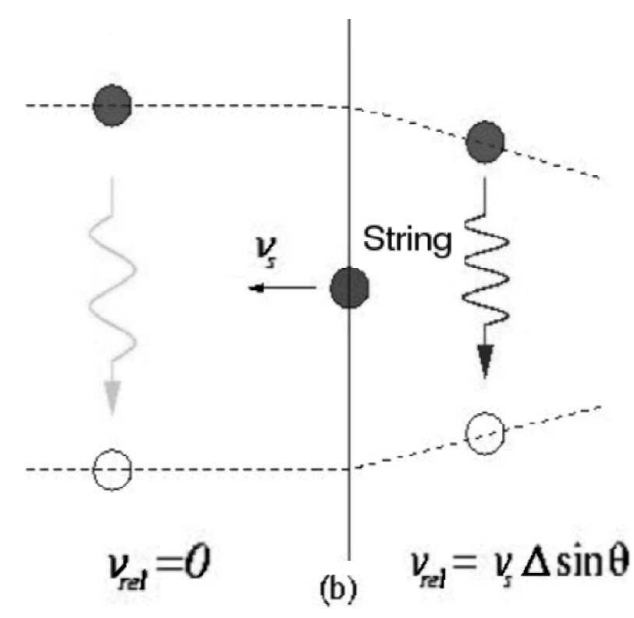

(b) $v_{\text {rel }}=v_{s} \Delta \sin \theta$

Figure 1-10: The Kaiser-Stebbins effect is originated from conic space-time around the cosmic string. A moving cosmic string induces relative speed between the light source and observer and causes a Doppler shift of photons. In the figure, $\Delta$ represents the effective deficit angle viewed from observer[26]. 
This phenomenon is generalised to junction of strings in next chapter (section 2.5) by summing the contribution of strings making the junction.

Beside space deformation, cosmic strings whenever they have wave train propagating on them can produce gravitation radiation.

\subsection{Gravitational radiation emitted by excited abelian cosmic strings}

Radiation of gravitational waves is a generic phenomenon by the time-varying fluctuation of the metric. They are tensor perturbations that correspond to the traceless and transverse parts of the metric perturbation : $h_{i}^{i}=0$ and $h_{j, i}^{i}=0$.

They have not been observed yet but some powerful telescope might be able to detect them in the future. Gravitational radiation comes predominantly from loop radiation : loops shrink and lose energy until they disappear. Though, we will focus more on radiation from straight cosmic strings coonfigurations and, later, we will study the junction case. Straight cosmic strings don't produce gravitational radiation since we need the interaction of both right and left mover to enable the possibility of an emission. This is because the stress energy tensor vanishes when there are only left or right movers, and the energy emitted is quadratic in the energy momentum tensor :

$$
T^{\mu \nu}(x)=\frac{\mu}{8 \pi} \int d u d v\left(a^{\prime \mu} b^{\prime \nu}+a^{\prime \nu} b^{\prime \mu}\right)
$$

where $\mathrm{u}$ and $\mathrm{v}$ correspond to right and left movers. In order to see the consequences of the interaction between right and left movers, we study strings attached to a wall and junctions in chapter 3 . 
The energy radiated by gravitational waves for frequency $\omega$ and momentum $\mathbf{k}$ per solid angle $\Omega$ :

$$
\frac{d E}{d \Omega}=2 G \int d \omega \omega^{2}\left[T^{\lambda \nu^{*}}(k) T_{\lambda \nu}(k)-\frac{1}{2}\left|T_{\lambda}^{\lambda}\right|^{2}(k)\right]
$$

In the case of a junction, we will have to sum the contribution of each of the strings forming the junction. An incomming wave on one of the string forming the junction will have a part reflected back at the junction and another part will go to the 2 other strings forming the junction. When left movers and right movers interact, we get a gravitational radiation emission. The formula (1.21) can then be rewritten in the following way :

$$
\frac{d E}{d \Omega}=\frac{G}{16 \pi^{2}} \sum_{i, j} \mu_{i} \mu_{j} \int d \omega \omega^{2}\left\{A_{i}^{*} \cdot A_{j} B_{i}^{*} \cdot B_{j}+A_{i}^{*} \cdot B_{j} B_{i}^{*} \cdot A_{j}-A_{i}^{*} \cdot B_{i}^{*} A_{j} \cdot B_{j}\right\}
$$

where . represents the dot product of quadrivectors. A (B) is a function depending on the outgoing (incomming) wave. The sum corresponds to summing the contribution of the different strings forming the junction. In the case where there is only right or left movers, A or B will vanish and there will be no gravitational power emitted. 


\title{
CHAPTER 2 \\ Lensing and CMB Anisotropies by Cosmic Strings at a Junction
}

In this chapter, we will generalise the previous properties of strings to the junction case. First, we find the metric, then we study the propagation of light phenomena and their consequences : Lensing and Kaiser-Stebbins effect.

The following is extracted from the article Lensing and CMB Anisotropies by Cosmic Strings at a Junction, published in Phys. Rev. D 77, 083502 (2008)

by : Robert Brandenberger, Hassan Firouzjahi, and Johanna Karouby

from : Physics Department, McGill University, Montreal, Canada

\begin{abstract}
The metric around straight arbitrarily-oriented cosmic strings forming a stationary junction is obtained at the linearized level. It is shown that the geometry is flat. The sum rules for lensing by this configuration and the anisotropies of the CMB are obtained.
\end{abstract}

Keywords : Cosmic strings, Cosmology 


\section{$2.1 \quad$ Introduction}

There has been a renewal of interests in cosmic strings [10]. This is partially due to the realization that in models of brane inflation cosmic strings, and not monopoles and domain walls, are copiously produced $[2,3]$. In these models of inflation, the inflaton is the distance between a D3-brane and an anti D3-brane [4]. There is an attractive force between the brane and anti-brane. Inflation ends when they collide and annihilate each other. The end product is a network of one-dimensional defects in the form of fundamental strings, F-strings, and D1-branes, D-strings. These can further combine to form a bound states of $\mathrm{p}$ F-strings and q D-strings, (p,q)-strings, for integers $\mathrm{p}$ and $\mathrm{q}$.

In more developed models of brane inflation, inflation happens in a warped region inside the string theory compactification [5]. This way, the scale of inflation as well as the effective tension of cosmic strings, $\mu$, are considerably smaller than the string scale [6]. One can easily saturate the current bounds on $G \mu$, the dimensionless number corresponding to the cosmic string tension [7].

When two $(p, q)$ cosmic strings intersect generally a junction is formed. This is due to charge conservation. This is in contrast to $\mathrm{U}(1)$ gauge cosmic strings. When two $U(1)$ gauge cosmic strings intersect, they usually exchange partners and intercommute with probability close to unity. In this view the formation of junctions may be considered a novel feature of the network of cosmic superstrings. Different

theoretical aspects of $(\mathrm{p}, \mathrm{q})$ strings construction were studied in $[8,9,10,23]$ while the cosmological evolution of a network of strings with junctions have been investigated in $[12]$. 
The most important cosmological implications of cosmic strings are the gravitational ones which are controlled by $G \mu$. Among them are the lensing effects. The geometry around a cosmic string is locally flat but globally it produces a deficit angle in the plane perpendicular to the string [13]. An observer looking at an object behind the string may see two identical images located on opposite sides of the string.

In this paper we study the lensing and CMB anisotropies due to an arbitrary configuration of straight cosmic strings forming a stationary junction. We will provide the sum rule for the formation of multiple images. The effects of cosmic string wakes on structure formation is briefly studied.

\subsection{The setup}

We are interested in the metric of strings at a stationary junction. We assume the junction is at rest. With an appropriate boost, one can also consider the case of a stationary junction moving with a constant velocity.

The action of $N$ semi-infinite strings joined at a point is

$$
S=-\sum_{i} \mu_{i} \int d t d \sigma \sqrt{-\left|\gamma_{i}\right|}
$$

where $\mu_{i}=\left|\vec{\mu}_{i}\right|$ is the tension of the i-th string (the vector pointing in direction of the string) and $\gamma_{i m n}$ is the metric induced on each string

$$
\gamma_{i m n}=g_{\mu \nu} \partial_{m} X_{i}^{\mu} \partial_{n} X_{i}^{\nu}
$$

Here $m, n=\{t, \sigma\}$ are the coordinates along the string worldsheet, $X^{\mu}$ are the space-time coordinates and $g_{\mu \nu}$ is the space-time metric. We shall choose $\sigma=0$ 
at the junction and increasing away from this point. Furthermore, $X^{0}=t$ while $X_{i}^{1}=x_{i}, X_{i}^{2}=y_{i}$ and $X_{i}^{3}=z_{i}$.

In order for the junction to be stationary, the vector sum of the tensions should vanish at the junction: $\sum_{i} \vec{\mu}_{i}=0$. Suppose the junction is the origin of the coordinate system and the unit vector along the i-th string is denoted by $\vec{n}_{i}$. The conditions for the junction to be stationary are translated into

$$
\sum_{i} \mu_{i} n_{i x}=\sum_{i} \mu_{i} n_{i y}=\sum_{i} \mu_{i} n_{i z}=0 .
$$

To solve the Einstein equations, we need to find the energy-momentum tensor $T^{\mu \nu}$ for the string configuration, obtained by varying the string action with respect to the metric

$$
\delta_{g} S=-\frac{1}{2} \sum_{i} \mu_{i} \int d t d \sigma \sqrt{-\gamma_{i}} \gamma_{i}^{m n} \partial_{m} X_{i}^{\mu} \partial_{n} X_{i}^{\nu} \delta g_{\mu \nu}=-\frac{1}{2} \int d^{4} x T^{\mu \nu} \delta g_{\mu \nu}
$$

For each string, one can choose $\sigma$ to represent the line element along the string

$$
d \sigma^{2}=d x_{i}^{2}+d y_{i}^{2}+d z_{i}^{2} \equiv d l_{i}^{2}
$$

which implies

$$
\gamma_{i 00}=1 \quad, \quad \gamma_{i_{\sigma \sigma}}=-1 \quad, \quad \gamma_{i 0 \sigma}=0 .
$$

Using these in Eq. (2.4) one obtains

$$
T^{\mu \nu}(\mathbf{x})=\sum_{i} \mu_{i} \int d l_{i} \gamma_{i}^{m n} \partial_{m} X_{i}^{\mu} \partial_{n} X_{i}^{\nu} \delta^{3}\left(\mathbf{x}-\mathbf{x}_{i}\right),
$$


where here and in the following, the boldface letters represent the spatial parts of the four-vectors $X^{\mu}$.

We start from a flat background metric $\eta_{\mu \nu}=\operatorname{diag}(1,-1,-1,-1)$. Up to linearized level, the metric is

$$
g_{\mu \nu}=\eta_{\mu \nu}+h_{\mu \nu}
$$

where $h_{\mu \nu}$ is the perturbation due to cosmic strings, $\left|h_{\mu \nu}\right|<<1$.

The linearized Einstein equations are

$$
R_{\mu \nu}=8 \pi G\left(T_{\mu \nu}-\frac{1}{2} \eta_{\mu \nu} T\right) \equiv 8 \pi G S_{\mu \nu},
$$

where $R_{\mu \nu}$ is the Ricci tensor and $T=T_{\mu}^{\mu}$ is the trace of the energy-momentum tensor. Furthermore, using (2.7) one obtains $S_{0 \mu}=0$ while

$$
S_{a b}(\mathbf{x})=\sum_{i} \mu_{i}\left(\delta_{a b}-n_{i a} n_{i b}\right) \int d l_{i} \delta^{3}\left(\mathbf{x}-\mathbf{x}_{i}\right),
$$

where $a, b$ are the spatial indices.

The Einstein equations as usual are subject to the choice of gauge. We use the harmonic gauge where

$$
h_{\nu, \mu}^{\mu}-\frac{1}{2} h_{\mu, \nu}^{\mu}=0
$$

and

$$
R_{\mu \nu}=-\frac{1}{2} \square h_{\mu \nu},
$$

where $\square$ is the four-dimensional Laplacian. 
The general solution of (2.9) and (2.12) is (for example see [24] )

$$
h_{\mu \nu}(\mathbf{x})=-4 G \int d^{3} \mathbf{y} \frac{S_{\mu \nu}(t-|\mathbf{y}-\mathbf{x}|, \mathbf{y})}{|\mathbf{y}-\mathbf{x}|}
$$

The term $t-|\mathbf{y}-\mathbf{x}|$ stands for the retarded time. For our case of a static junction we are interested in the time-independent solution.

Using (2.10) in (2.13) yields $h_{0 \mu}=0$ while

$$
h_{a b}(\mathbf{x})=-4 G \sum_{i} \mu_{i}\left(\delta_{a b}-n_{i a} n_{i b}\right) \int_{0}^{\infty} \frac{d l_{i}}{\left|\mathbf{x}-\mathbf{x}_{i}\right|} .
$$

Knowing that along each string $\mathbf{x}_{i}=l_{i} \vec{n}_{i}$, the above integral can be calculated, and our final solution is

$$
h_{a b}=4 G \sum_{i} \mu_{i}\left(\delta_{a b}-n_{i a} n_{i b}\right) \ln \left(\frac{r-\vec{r} \cdot \vec{n}_{i}}{r_{0}}\right) \text {, }
$$

where $r=|\mathbf{x}|$ is measured from the point of the junction and $r_{0}$ is a constant of integration. One can directly check that the solution (2.15) satisfies the harmonic gauge (2.11).

Similar to what happens in the case of a single infinite string, at points on each string where $\vec{r}=r \vec{n}_{i}$, the metric is singular. This is because we have started with delta function sources. In the realistic situation when the strings have finite width, this singularity is smoothed out [16]. On the other hand, at points on the opposite side of each string where $\vec{r}=-r \vec{n}_{i}$, the metric is non-singular.

Our solution in Eq. (2.15) is valid for any arbitrary configuration of straight cosmic strings in a stationary junction. In order to understand its general applicability, let us consider the case of a single infinite straight string, the case originally 
considered by Vilenkin [13]. This configuration in our formalism corresponds to two semi-infinite strings with equal tension extended back to back [15] with $\vec{n}_{1}=-\vec{n}_{2}$. We may choose the strings to extended oppositely along the $z$-axis. We obtain $h_{z z}=h_{z x}=h_{z y}=h_{x y}=0$, while

$$
\begin{aligned}
h_{x x}=h_{y y} & =4 G \mu \ln \left(\frac{r^{2}-\left(r \cdot \vec{n}_{1}\right)^{2}}{r_{0}^{2}}\right) \\
& =8 G \mu \ln \left(\frac{r_{\perp}}{r_{0}}\right) .
\end{aligned}
$$

But $r_{\perp}$ is the normal distance to the string from the point of the observer at position $\mathbf{x}$, and we obtain Vilenkin's solution [13].

\subsection{The flatness of the geometry}

The geometry around an infinite cosmic string is flat away from the string core. One may ask whether or not this is also true in our case of cosmic strings at a static junction. At first sight, the metric given in (2.15) does not seem to be flat. To address this question we need to calculate the components of the Riemann tensor. Since the time coordinate decouples from our solution, effectively we are dealing with a three-dimensional spatial geometry. In three dimensions, both the Riemann tensor $R_{a b c d}$ and the Ricci tensor $R_{a b}$ have 6 independent components. This indicates that the components of Riemann tensor can be expressed in terms of Ricci tensor. More explicitly

$R_{x z z y}=R_{x y} \quad, \quad R_{x y y z}=R_{x z} \quad, \quad R_{y x x z}=R_{y z} \quad, \quad R_{x y x y}=\frac{1}{2}\left(R_{z z}-R_{x x}-R_{y y} \chi_{2}\right.$

while the remaining two components $R_{x z x z}$ and $R_{y z y z}$ are obtained by the appropriate permutations of the $x, y$ and $z$ coordinates. 
Our solution is a vacuum solution with the strings as sources. It is clear from (2.9) that $R_{a b}=0$ away from the strings. Using this in (2.17) we can immediately conclude that all components of the Riemann tensor vanish and the geometry given by the metric (2.15) is indeed flat.

The fact that the junction is stationary is the crucial requirement for the flatness of the geometry. It is evident that for non-stationary junctions the space-time is curved. The extent of the departure from a flat geometry is directly controlled by the extent of violation of the stationarity conditions. For example, using the metric (2.15) one can show that

$$
R_{x y x y}=\frac{-2 G}{r^{3}} \sum_{i} \mu_{i}\left(n_{i x} x+n_{i y} y-n_{i z} z\right) .
$$

This is zero due to the force balance conditions (2.3).

\subsection{The Propagation of Light}

One of the novel cosmological features of cosmic string is the lensing effect. The metric of a straight cosmic string is locally flat. But globally the geometry around the string has a deficit angle given by $\Delta=8 \pi G \mu$. This results in the formation of two identical images of an object located behind the string. When looking at an object located behind a static junction of semi-infinite strings, one naturally expects multiple images to form.

The lensing by three co-planar strings forming a Y-shaped junction was studied by Shlaer and Wyman [5]. An observer looking at an object, say a galaxy, located

behind the plane of the strings will see three (identical) images; one image is the object itself and the other two are its lensing counterparts. 


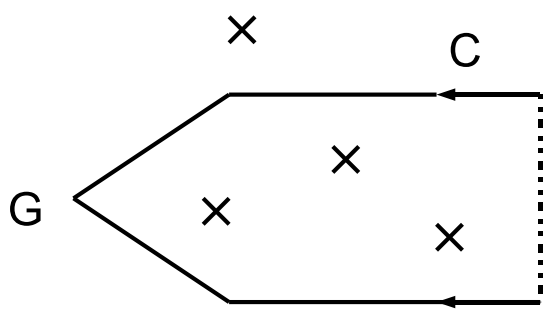

Figure 2-1: In this figure two parallel light rays emitted from infinity are deflected towards the point $\mathrm{G}$. The contour $\mathrm{C}$ is made of the point $\mathrm{G}$, the light rays and the line connecting the two light rays distance at infinity, denoted by the dashed line. The strings cross the plane spanned by the contour at the points indicated by the cross signs. Only those strings which are enclosed by the contour contribute in (2.19) and $(2.25)$.

In the method used in [5], one starts with three infinite strings intersecting at the point of the junction. This will produce two $\mathbf{Y}$-shaped junctions oriented oppositely at the junction. One can "cut-and-paste" one junction and keep the remaining one. This method will correctly produce the lensing by the junction, as demonstrated in $[5]$.

In this section we would like to consider the general case of an arbitrary number of stationary cosmic strings forming a junction, co-planar or not, and study the resulting lensing phenomena in cosmology.

Suppose two parallel light rays are emitted from infinity towards the junction. Once the line connecting the light rays passes a string, the light rays are expected to be bent towards each-other. Suppose the light rays stay co-planar and meet at a point, say G. The difference in the velocity vectors at the point of intersection G can be obtained by the method of parallel transportation around a closed curved C. 
The curve $\mathrm{C}$ is composed of the point $\mathrm{G}$, the two light rays from infinity and the line connecting these rays at infinity. For a schematic view see Fig. 2-1. The difference in the velocity vectors at $G$ is [18]

$$
\delta v^{\alpha}=-\frac{1}{2} \int_{S} R_{\beta \gamma \lambda}^{\alpha} v^{\beta} d x^{\gamma} \wedge d x^{\lambda},
$$

where the integration is over the closed surface S bounded by $\mathrm{C}$.

Of course, in the empty regions away from strings the Riemann tensor vanishes as we shown before and the above integral is zero, as expected. However, when the surface intersects strings the Riemann tensor provides delta-function contributions and the integral does not vanish. This demonstrates that the relative change of the velocity vectors and consequently the lensing effects are directly controlled by the number and the orientation of the cosmic strings which intersect the surface S. In the spirit, the method is analogous to the residue theorem for the integration of an analytical function in the complex plane.

Without loss of generality, we may suppose that the light rays are emitted along the negative z-direction in the y-z plane. Using Eq. (2.10) one obtains

$$
S_{a b}=\sum_{i} \frac{\mu_{i}}{n_{i x}}\left(\delta_{a b}-n_{i a} n_{i b}\right) \delta\left(y-y_{i}\right) \delta\left(z-z_{i}\right)
$$

where $y_{i}$ and $z_{i}$ are the coordinates of the point of intersection of the i-th string with the y-z plane. The factor $n_{i x}$ in the denominators originates from replacing $d l_{i}$ along the string by $d x$ via $d x=n_{i x} d l_{i}$. 
Using this in Eq. (2.19) one obtains

$$
\begin{aligned}
\delta v^{x} & =\int d y d z R_{x z z y}=\int d y d z R_{x y}=8 \pi G \int d y d z S_{x y} \\
& =-8 \pi G \sum_{i} \mu_{i} n_{i y}
\end{aligned}
$$

where the sum is over those strings which intersect the plane of the light rays (the y-z plane here) and are enclosed by the surface $\mathrm{S}$ bounded by the light rays.

Similarly, for the change of velocity in the $y$-direction one obtains

$$
\begin{aligned}
\delta v^{y} & =\int d y d z R_{y z z y}=\frac{1}{2} \int d y d z\left(R_{y y}+R_{z z}-R_{x x}\right) \\
& =8 \pi G \sum_{i} \mu_{i} n_{i x}
\end{aligned}
$$

where to obtain the last equation, the identity $n_{i x}^{2}+n_{i y}^{2}+n_{i x}^{2}=1$ has been used.

Finally, from (2.19) one can easily see that $\delta v_{z}=0$, which implies that there is no change of velocity in the direction tangential to the initial light rays.

Combining these results, one obtains the following coordinate independent representation of the change in velocity

$$
\delta \vec{v}=-8 \pi G \vec{k} \times \sum_{i} \vec{\mu}_{i}
$$

where the unit vector $\vec{k}$ represents the direction of the light rays at infinity (line of sight).

The angle between the two light rays at the point of intersection is

$$
\Delta=|\delta \vec{v}|=8 \pi G \mu_{e f f}
$$


where

$$
\mu_{e f f}=\left|\vec{k} \times \sum_{i} \vec{\mu}_{i}\right|
$$

As explained before, the sum is over those strings which intersect the plane S formed by the light rays up to their point of intersection. For the case of a single string with tension $\mu$ enclosed between the light rays, $\mu_{e f f}=\mu$ and we obtain the standard result for the deficit angle.

Now let us try to apply Eq. (2.24) to some examples. The first interesting example is the lensing by a $\mathbf{Y}$-shaped junction studied in [5]. The strings are in the $\mathrm{x}-\mathrm{y}$ plane and the light rays are emitted along the $\mathrm{z}$-direction. A schematic view of this situation is presented in Fig. 2-2 . Starting with the object located at point $A$ and moving in counterclockwise direction, we enclose the string with tension $\mu_{1}$ and $\mu_{e f f}=\mu_{1}$. Consequently, there is an image at point $A_{1}$ separated from $A$ by the deficit angle $8 \pi G \mu_{1}$. Continuing further counterclockwise, we enclose a second string (with tension $\mu_{2}$ ) and $\mu_{e f f}=\left|\vec{\mu}_{1}+\vec{\mu}_{2}\right|$. Because of the force balance condition this is the same as $\mu_{3}$. So the second image is at the point $A_{2}$ which is separated from the object $A$ by a deficit angle $8 \pi G \mu_{3}$. Finally, continuing further to enclose the third string, the effective tension vanishes due to force balance condition $\overrightarrow{\mu_{1}}+\overrightarrow{\mu_{2}}+\overrightarrow{\mu_{3}}=0$ and there is no other image. This means that the points $A, A_{1}$ and $A_{2}$ form a triangle. This has an interesting geometrical interpretation: Each image acts as a source for the other two images. The object $A$ is the source for $A_{1}$ through the string $\mu_{1}$, the image $A_{1}$ acts as source for $A_{2}$ via the string $\mu_{2}$ and the image $A_{2}$ 
is the source for $A$ via the string $\mu_{3}$. This is in exact agreement with the prescription provided for a $\mathbf{Y}$-shaped junction in [5].

In this example the strings are between the observer and the object and the plane of the strings is perpendicular to the line of sight. Whether or not the observer actually sees an image depends on the angular distances between each string and the object. If a distance is larger than the deficit angle, then the corresponding image is not observable.

One can generalize the example of the $\mathbf{Y}$-shaped junction to the case of $N$ coplanar cosmic strings at junction. As before, the plane of the strings is between the object and the observer and is perpendicular to the line of sight. Following the same steps as above, we obtain $N-1$ images plus the object itself. This set of $N$ points forms a closed loop due to the force balance condition. For each image, the effective tension is the magnitude of the vector sum of all strings enclosed between the object and the corresponding image. Geometrically, as before, this means that each image is the source for the nearby images via the enclosed string. Starting with the object $A$, the chain of object $\rightarrow$ image is given by $A \rightarrow A_{1} \rightarrow A_{2} \rightarrow . . \rightarrow A_{N-1} \rightarrow A$. The schematic view of this case is presented in Fig. 2-2.

Another interesting case is when the light rays are parallel to the plane of the co-planar strings. Now the strings are located in the $\mathrm{y}-\mathrm{z}$ plane and two light rays are emitted along the z-direction. If the light rays are on the same side of the plane, then no string is enclosed by them and the light rays will stay parallel. Now suppose the light rays are emitted from opposite sides of the plane. To simplify the analysis suppose the light rays are in the $\mathrm{x}-\mathrm{z}$ plane so they are emitted at $\left(x_{1}, y_{1}, \infty\right)$ and 

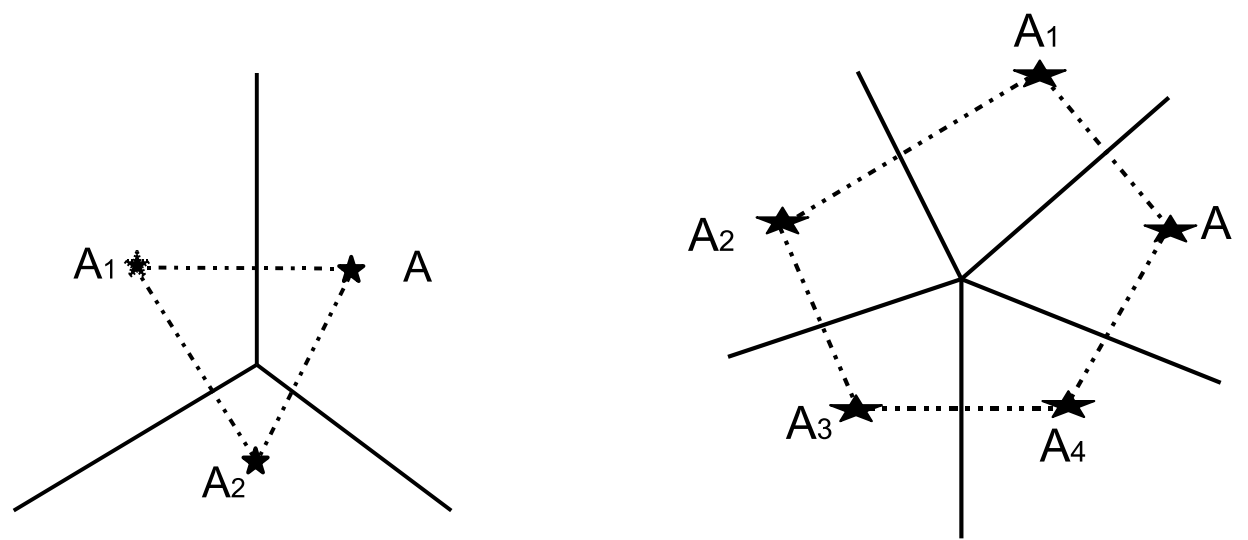

Figure 2-2: In this figures the multiple lensings by $N$ co-planar strings at a junction is sketched. The object $\mathrm{A}$ and its lensing counterparts $A_{i}, i=1 . . N-1$, form a closed loop. Each image is the source for the nearby images via the enclosed string. For example, in the three-string junction in the left figure, the object $\mathrm{A}$ is the source for the image $A_{1}$ via the string $\mu_{1}, A_{1}$ is the source for the image $A_{2}$ via the string $\mu_{2}$ and $A_{2}$ is the source for the object $\mathrm{A}$ via the string $\mu_{3}$.

$\left(x_{2}, y_{1}, \infty\right)$ with $x_{1} x_{2}<0$. We already know that $\delta v_{z}=0$, i.e. there is no change of velocity in the direction parallel to the light rays. Also, from Eq. (2.22) one obtains $\delta v_{y}=0$ because the strings have no $n_{x}$ components. On the other hand, from Eq. (2.21) one obtains

$$
\left|\delta v_{x}\right|=8 \pi G\left|\sum_{i} \mu_{i} n_{i y}\right|
$$

where the sum is over the strings in the upper part of the y-z plane. From the force balance condition, this is also equivalent to the sum over the strings in the lower part of the $\mathrm{y}-\mathrm{z}$ plane. Interestingly enough, we see that the light rays which start out parallel to the plane of strings are now deflected towards the plane. 


\subsection{CMB anisotropies and cosmic String wakes}

The effects of moving string on CMB anisotropy were studied by Kaiser and Stebbins [19]. These authors showed that as a consequences of the conical structure of space perpendicular to the string, a moving string will produce a line discontinuity in temperature anisotropy maps, with the amplitude of the discontinuity given by

$$
\frac{\delta T}{T}=G \mu \gamma(v) v
$$

where $v$ is he string velocity perpendicular to our line of sight towards the string, and $\gamma(v)$ is the Lorentz factor. The effect is due to the relative Doppler shift in the photons passing on the two sides of the string. The Kaiser-Stebbins effect is a key distinctive observational signature for strings. Methods to search for this signature have recently been discussed in [20, 21] (see also [22] for older work).

One may naturally ask how this can be generalized to the case of strings at a junction when the junction is moving with a constant velocity $v$. It is understood that the junction and the strings attached to it move as a solid object and the no force condition (2.3) still holds.

We are interested in change in the observed frequency between two parallel light rays (with the same initial frequency) in the presence of the moving junction. As before, we take the light rays to be moving in the $y-z$ plane, and initially along the z-direction. In terms of Eq. (2.19) instead of $v^{\mu}$ we use the momentum four-vector $p^{\mu}=(E, \mathbf{p})$, where $E=|\mathbf{P}|=\hbar \omega$. This yields

$$
\delta \omega=\omega \int R_{0 z z y} d y d z
$$


Unlike in the case of a static junction, the Riemann tensor has non-zero components like $R_{t a b c}$ and $R_{t a t b}$ due to the string motion. In 4-D space-time the Riemann tensor has more components than the Ricci tensor does. However, we can go to the junction's rest frame where the space-time is static and our results from the previous section can be used readily. Denote the coordinate system where the junction is static by $x^{\mu^{\prime}}$ while the coordinate system used by the observer and the light sources is represented by $x^{\mu}$. We have

$$
R_{0 z z y}=\frac{\partial x^{\alpha^{\prime}}}{\partial t} \frac{\partial x^{\beta^{\prime}}}{\partial z} \frac{\partial x^{\mu^{\prime}}}{\partial z} \frac{\partial x^{\nu^{\prime}}}{\partial y} R_{\alpha^{\prime} \beta^{\prime} \mu^{\prime} \nu^{\prime}}
$$

where now $R_{\alpha^{\prime} \beta^{\prime} \mu^{\prime} \nu^{\prime}}$ has no time-like indices.

In general the junction velocity vector $\vec{v}$ can have components in $\mathrm{x}, \mathrm{y}$ and $\mathrm{z}-$ directions. Suppose the junction is moving along the $\mathrm{x}$-direction. Then one obtains

$$
\begin{aligned}
\frac{\delta \omega}{\omega} & =\left|\int d y^{\prime} d z^{\prime} \frac{\partial x^{\prime}}{\partial t} R_{x^{\prime} z^{\prime} z^{\prime} y^{\prime}}\right| \\
& =8 \pi G \gamma v\left|\sum_{i} \mu_{i} n_{i y}\right| .
\end{aligned}
$$

To obtain the final line, the same procedure as in Eq. (2.21) has been used. As before, in the above the sum is over the strings which are enclosed by the light rays. Similarly, in the case when the junction is moving along the y-direction one obtains

$$
\begin{aligned}
\frac{\delta \omega}{\omega} & =\left|\int d y^{\prime} d z^{\prime} \frac{\partial y^{\prime}}{\partial t} R_{y^{\prime} z^{\prime} z^{\prime} y^{\prime}}\right| \\
& =8 \pi G \gamma v\left|\sum_{i} \mu_{i} n_{i x}\right|
\end{aligned}
$$


where to obtain the final result Eq. (2.22) was used.

Finally, if the junction is moving along the z-direction parallel to the light arrays one can show that $\delta \omega$ vanishes.

Combining all these results, one can show that

$$
\frac{\delta \omega}{\omega}=8 \pi G \gamma\left|\vec{v} \cdot\left(\vec{k} \times \sum_{i} \vec{\mu}_{i}\right)\right|
$$

where as before $\vec{k}$ represents the line of sight and the sum is over the strings which are enclosed by the light rays. Interestingly enough, this formula has the same functional form as that of the a single string [23].

The implications of Eq. (2.32) for the CMB anisotropies are parallel to those of Eq. (2.24) for lensing. Each string at the junction produces its own CMB anisotropies. The change in the CMB temperature across the $\mathrm{i}$-th string is given by

$$
\frac{\delta T}{T}=8 \pi G \gamma\left|\vec{v} \cdot\left(\sum_{i} \vec{\mu}_{i} \times \vec{k}\right)\right| .
$$

Interestingly enough, the temperature anisotropy is different for different legs of the strings at the junction, depending on the tensions and orientations of the strings.

We conclude that the distinctive signature of strings with junctions for CMB anisotropies is the possibility of line discontinuities joined at a point for an observer looking at the surface of last scattering. The details of this picture depends on the orientations of the strings and the direction of its motion of the junction with respect to the line of sight. In recent work [20], the Canny algorithm [24], an edge detection algorithm, was shown to yield a sensitive statistic with which CMB line 
discontinuities can be identified in the sky. Since string junctions are a prediction of cosmic superstrings (simple gauge theory strings do not admit junctions), it would be interesting to develop modified edge detection algorithms which can differentiate between strings with and without junctions in CMB anisotropy maps. Work on this issue is in progress.

The lensing produced by the cosmic string deficit angle also produces distinctive signatures for structure formation, namely "wakes" [25]. For the rest of this section, we will briefly discuss the implications of string junctions for cosmic string wakes. Thus, we consider the accretion of matter by a moving junction.

Consider two massive non-relativistic objects at rest in the frame of the cosmic microwave background in the presence of a moving junction. As in the previous section, it is convenient to go to the rest frame of the junction. In this frame, the two massive objects are moving towards the junction. When the line connecting the objects passes any string, the objects are attracted towards each other behind the cosmic string. This can provide a mechanism for structure formation by string wakes $[25,26,27]$.

In the case of a single infinite string, the lensing of matter behind a moving string leads to a region of twice the background density in the wake of the moving string. The opening angle of the wake is given by the deficit angle. The question we wish to address in this section is how the wake structure generalizes to strings with junctions.

The results obtained in Section (IV) for the bending of two light rays can be used for massive objects too. The only change is the addition of $\gamma v$ factor due to the 
change of frame. The relative velocity between the massive objects at the intersection is

$$
\delta \vec{v}=-8 \pi G \gamma v \vec{k} \times \sum_{i} \vec{\mu}_{i}
$$

On the other hand, the angle between the particles trajectories is $\Delta_{m}=|\delta \vec{v}| / v$, and

one obtains $\Delta_{m}=8 \pi G \gamma\left|\vec{k} \times \sum_{i} \vec{\mu}_{i}\right|$. This means that around each leg of the string junction there is a wedge-shaped wake with opening angle $\Delta_{m}$. In each of these regions, the matter density is doubled, i.e. $\delta \rho / \rho=2$.

Since the cosmic strings cannot be the dominant source of structure formation, and since string wakes undergo non-trivial non-linear evolution, it will presumably be more difficult to find distinctive signatures for string junctions in large-scale structure surveys than in CMB anisotropy maps. In principle, topological statistics such as Minkowski functionals [28] have the power to find such signatures [29].

\subsection{Conclusions}

Working at the level of linearized gravity, we have derived the metric of a static string junction, with an arbitrary number of strings joining. We have shown that away from the world sheet of the strings, the metric is flat. Thus, the geometry generalizes that for a single straight infinitely long string. Each string segment produces a deficit angle, and thus deflects both light and matter. We have derived the lensing of light by a string junction, discussed the CMB anisotropies produced by a string junction which is in uniform motion relative to the frame of the microwave background, and commented on the formation of string wakes. 
We have identified a junction of line discontinuities in CMB anisotropy maps as a distinctive signature of strings with junctions, and proposed that one could look for these signatures by a generalized edge detection algorithm. Finding positive evidence for such string segments would provide a boost for superstring theory, since it is in the context of superstring theory that the existence of strings with junctions

first came to prominence. Strings with junctions are quite generic in the context of superstring theory, but they do not appear in simple gauge field theory models of cosmic strings.

\subsection{Acknowledgments}

We would like to thank K. Dasgupta, O. Saremi and H. Tye for useful discussions. This work is supported by NSERC, the Canada Research Chair Program and an FQRNT Team Grant. 


\section{REFERENCES}

[1] T. W. B. Kibble, "Cosmic strings reborn?," astro-ph/0410073.

[2] S. Sarangi and S.-H. H. Tye, "Cosmic string production towards the end of brane inflation," Phys. Lett. B 536, 185 (2002), hep-th/0204074.

[3] N. T. Jones, H. Stoica and S.-H. H. Tye, " The production, spectrum and evolution of cosmic strings in brane inflation", Phys. Lett. B 563, 6 (2003), hepth/0303269.

[4] G. R. Dvali and S. H. H. Tye, "Brane inflation," Phys. Lett. B 450, 72 (1999), hep-ph/9812483;

C. P. Burgess, M. Majumdar, D. Nolte, F. Quevedo, G. Rajesh and R. J. Zhang, "The inflationary brane-antibrane universe," JHEP 0107, 047 (2001), hepth/0105204;

G. R. Dvali, Q. Shafi and S. Solganik, "D-brane inflation,", hep-th/0105203.

[5] S. Kachru, R. Kallosh, A. Linde, J. M. Maldacena, L. McAllister and S. P. Trivedi, "Towards inflation in string theory," JCAP 0310, 013 (2003) hep-th/0308055;

H. Firouzjahi and S. H. H. Tye, "Closer towards inflation in string theory," Phys. Lett. B 584, 147 (2004) hep-th/0312020;

C. P. Burgess, J. M. Cline, H. Stoica and F. Quevedo, "Inflation in realistic Dbrane models," JHEP 0409, 033 (2004) hep-th/0403119.

[6] H. Firouzjahi and S. H. Tye, "Brane inflation and cosmic string tension in superstring theory," JCAP 0503, 009 (2005) [arXiv:hep-th/0501099].

[7] M. Wyman, L. Pogosian and I. Wasserman, "Bounds on cosmic strings from WMAP and SDSS," Phys. Rev. D 72, 023513 (2005) [Erratum-ibid. D 73, 089905 (2006)] [arXiv:astro-ph/0503364];

F. A. Jenet et al., "Upper bounds on the low-frequency stochastic gravitational wave background from pulsar timing observations: Current limits and future prospects," astro-ph/0609013;

N. Bevis, M. Hindmarsh, M. Kunz and J. Urrestilla, "Fitting CMB data with cosmic strings and inflation," arXiv:astro-ph/0702223. 
[8] E. J. Copeland, R. C. Myers and J. Polchinski, "Cosmic F- and D-strings," JHEP 0406, 013 (2004), hep-th/0312067;

L. Leblond and S.-H. H. Tye, "Stability of D1-strings inside a D3-brane," JHEP 0403, 055 (2004), hep-th/0402072.

[9] H. Firouzjahi, L. Leblond and S. H. Henry Tye, "The (p,q) string tension in a warped deformed conifold," JHEP 0605, 047 (2006) hep-th/0603161;

H. Firouzjahi, "Dielectric (p,q) strings in a throat," JHEP 0612, 031 (2006) hepth/0610130;

K. Dasgupta, H. Firouzjahi and R. Gwyn, "Lumps in the throat," JHEP 0704, 093 (2007) [arXiv:hep-th/0702193].

[10] M. G. Jackson, N. T. Jones and J. Polchinski, "Collisions of cosmic F- and Dstrings," JHEP 0510, 013 (2005), hep-th/0405229;

A. Hanany and K. Hashimoto, "Reconnection of colliding cosmic strings," JHEP 0506, 021 (2005) [arXiv:hep-th/0501031];

K. Hashimoto and D. Tong, "Reconnection of non-abelian cosmic strings," JCAP 0509, 004 (2005) [arXiv:hep-th/0506022].

[11] E. J. Copeland, T. W. B. Kibble and D. A. Steer, "Collisions of strings with Y junctions," Phys. Rev. Lett. 97, 021602 (2006), hep-th/0601153;

E. J. Copeland, T. W. B. Kibble and D. A. Steer, "Constraints on string networks with junctions,' Phys. Rev. D 75, 065024 (2007) [arXiv:hep-th/0611243].

[12] S. H. Tye, I. Wasserman and M. Wyman, "Scaling of multi-tension cosmic superstring networks," Phys. Rev. D 71, 103508 (2005) [Erratum-ibid. D 71, 129906 (2005)] [arXiv:astro-ph/0503506];

L. Leblond and M. Wyman, "Cosmic necklaces from string theory," astro$\mathrm{ph} / 0701427$;

A. Avgoustidis and E. P. S. Shellard, "Velocity-Dependent Models for NonAbelian/Entangled String Networks," arXiv:0705.3395 [astro-ph];

A. Rajantie, M. Sakellariadou and H. Stoica, "Numerical experiments with p Fand q D-strings: the formation of (p,q) bound states," arXiv:0706.3662 [hep-th].

[13] A. Vilenkin, "Gravitational Field Of Vacuum Domain Walls And Strings," Phys. Rev. D 23, 852 (1981).

[14] S. Weinberg, "Gravitation and cosmology: principles and applications of the general theory of rel Wiley, New York, 1972. 
[15] J. D. Jackson, "Classical Electrodynamics," John Wiley \& Sons, 2001.

[16] R. Gregory, "Gravitational stability of local strings," Phys. Rev. Lett. 59, 740 (1987).

[17] B. Shlaer and M. Wyman, "Cosmic superstring gravitational lensing phenomena: Predictions for networks of (p,q) strings," Phys. Rev. D 72, 123504 (2005) [arXiv:hep-th/0509177].

[18] C. Misner, K. Thorne and J. Wheeler. "Gravitation,"

[19] N. Kaiser and A. Stebbins, "Microwave Anisotropy Due To Cosmic Strings," Nature 310, 391 (1984).

[20] S. Amsel, J. Berger and R. H. Brandenberger, "Detecting Cosmic Strings in the CMB with the Canny Algorithm," arXiv:0709.0982 [astro-ph].

[21] A. S. Lo and E. L. Wright, "Signatures of cosmic strings in the cosmic microwave background," arXiv:astro-ph/0503120;

E. Jeong and G. F. Smoot, "The Validity of the Cosmic String Pattern Search with the Cosmic arXiv:astro-ph/0612706;

E. Jeong and G. F. Smoot, "Search for cosmic strings in CMB anisotropies," Astrophys. J. 624, 21 (2005) [arXiv:astro-ph/0406432].

[22] R. Moessner, L. Perivolaropoulos and R. H. Brandenberger, "A Cosmic string specific signature on the cosmic microwave background," Astrophys. J. 425, 365 (1994) [arXiv:astro-ph/9310001].

[23] T. Vachaspati, "Gravitational Effects of Cosmic Strings," Nucl. Phys. B 277, 593 (1986).

[24] J. Canny, "A computational approach to edge detection", IEEE Trans. Pattern Analysis and Machine Intelligence 8, 679 (1986).

[25] J. Silk and A. Vilenkin, "Cosmic Strings And Galaxy Formation," Phys. Rev. Lett. 53, 1700 (1984).

[26] A. Stebbins, S. Veeraraghavan, R. H. Brandenberger, J. Silk and N. Turok, "Cosmic String Wakes," Astrophys. J. 322, 1 (1987).

[27] A. Sornborger, R. H. Brandenberger, B. Fryxell and K. Olson, "The structure of cosmic string wakes," Astrophys. J. 482, 22 (1997) [arXiv:astro-ph/9608020]. 
[28] J. Schmalzing, M. Kerscher and T. Buchert, "Minkowski functionals in cosmology," arXiv:astro-ph/9508154.

[29] H. Trac, D. Mitsouras, P. Hickson and R. H. Brandenberger, "Topology of the Las Campanas Redshift Survey," Mon. Not. Roy. Astron. Soc. 330, 531 (2002) [arXiv:astro-ph/0007125]. 


\section{CHAPTER 3}

\section{Gravitational Radiation by Cosmic Strings in a Junction}

In this chapter, we will study another property of strings : The gravitational radiation emission. We first study the simple case of a semi-string attached to a wall and then generalise it to the junction case.

The following is extracted from the article Gravitational radiation by cosmic strings in a junction, published in JCAP01(2009)008 .

by : Robert Brandenberger ${ }^{a, b}$, Hassan Firouzjahi ${ }^{c}$, Johanna Karouby ${ }^{a}$ and Shahram $\mathrm{Khosravi}^{d, e}$

from : ${ }^{a}$ Physics Department, McGill University, Montreal, Canada

${ }^{b}$ Institute of High Energy Physics, Chinese Academy of Sciences,Beijing, China

${ }^{c}$ School of Physics, Institute for Research in Fundamental Sciences (IPM), Tehran,Iran.

${ }^{d}$ School of Astronomy,IPM, Tehran, Iran.

${ }^{e}$ Physics Department, Faculty of Science, Tarbiat Mo'alem University, Tehran, Iran 
Abstract : The formalism for computing the gravitational power radiation

from excitations on cosmic strings forming a junction is presented and applied to the simple case of co-planar strings at a junction when the excitations are generated along one string leg. The effects of polarization of the excitations and of the backreaction of the gravitational radiation on the small scale structure of the strings are studied.

Keywords: Gravity Waves, Cosmic Strings

\subsection{Introduction}

In models of brane inflation cosmic strings are produced (for a review see [16]). This has led to a revival of interest in cosmic strings (see e.g. $[10,15,4]$ ). Cosmic strings forming in the context of brane models can take the form of Fundamental strings (F-strings), D1-branes (D-strings) or their bound states, ((p,q) strings). A $(\mathrm{p}, \mathrm{q})$ string is a bound state of $p$ F-strings and $q$ D-strings. Networks of stringy cosmic strings which can involve strings with different values of $p$ and $q$ have features unlike those of simple gauge theory strings. Unlike U(1) gauge theory cosmic strings which inter-commute when they intersect, in the case of cosmic $(p, q)$ strings there are conservation laws which prevent the inter-commutation of strings with different values of $p$ and $q$. Instead, a string junction can be formed. For example, a $p$ string and a $q$ string can join at a junction to form a $(\mathrm{p}, \mathrm{q})$ string. The construction of cosmic strings with junctions and its cosmological implications were studied in $[22,23,5,8,9,12,14,15,16,13,11,10]$. 
Gravitational wave (GW) emission from loops and cusps of cosmic strings has been studied (for a comprehensive review see $[17,13]$ and for more recent analyses see $[19,20])$. A straight infinite string does not emit GW. This is because to emit GW, as we shall explicitly see in the next section, both left-movers and right-movers should be present on the string world sheet. In a network of cosmic strings, it is quite natural to expect that wiggles of different wavelengths are generated on the world sheet of an infinite string. These wiggles, for example, are left over from times when the correlation length of the string network was much smaller, or are remnants of string inter-commutations which took place in the past. These wiggles cause the GW emission from long strings and can smooth out the wiggles of the string world sheet. GW emission from wiggles on a straight string were studied in [21, $22,27]$. In particular, in [27] left-moving and right-moving wave-trains of different wavelengths and amplitudes on an infinite string were considered. It was shown that when the wavelengths and the amplitudes of the wave-trains are comparable, the GW emission is mainly from lower harmonics and is proportional to the frequency of the wave-trains. This indicates that excitations of higher frequency die out faster than excitations of shorter frequencies. On the other hand, when the wavelengths and amplitudes of the wave-trains are much different, then GW emission is exponentially suppressed.

In this paper, we consider gravitational radiation form strings at a junction. As we shall see, the presence of the junction leads to mixing of left and right-moving excitations on the string which is the necessary criterium for the emission of GW. In Section 2, we present the setup of our study. In Section 3 we study three examples. 
The first example is GW emission from a semi-infinite string attached to a rigid wall. The second example corresponds to GW emission from a stationary junction. The third example concerns GW emission from a non-stationary junction. As we shall see, the expressions for the gravitational wave power radiated has a similar form in all three examples. We discuss our results and summarize our conclusions in Section 4.

\subsection{The Setup}

Our setup consists of semi-infinite strings forming a stationary junction. The formalism in this section is valid for any number of semi-infinite strings meeting at a junction. However, to be specific, in our study we shall focus on the simple example where three semi-infinite strings form a stationary junction.

The world-sheet of each string is described by a temporal coordinate $\tau$ and a string length parameter $\sigma$. The induced metric $\gamma_{i a b}$ on each string is given by

$$
\gamma_{i a b}=g_{\mu \nu} \partial_{a} X_{i}^{\mu} \partial_{b} X_{i}^{\nu}
$$

Here and in the following, we reserve $\{a, b\}=\{\tau, \sigma\}$ for the string world-sheet indices while Greek indices represent the four-dimensional space-time coordinates. Furthermore, $X_{i}^{\mu}$ stands for the position of the $i$-th string in four space-time dimensions.

We impose the conformal temporal gauge on the string world-sheet for which $X_{i}^{0}=t=\tau$ and $\gamma_{i 0 \sigma}=0$. This is equivalent to

$$
\dot{\mathbf{x}}_{i} \cdot \mathbf{x}_{i}^{\prime}=0 \quad, \quad \dot{\mathbf{x}}_{i}^{2}+\mathbf{x}_{i}^{\prime 2}=1
$$


Here an overdot and a prime denote derivatives with respect to $t$ and $\sigma$, respectively, while $\mathbf{x}_{i}$ represent the spatial components of the $i$-th string

For the components of the induced metric on the string world sheet we obtain

$$
\gamma_{i 00}=1-\dot{\mathbf{x}}_{i}^{2} \quad, \quad \gamma_{i \sigma \sigma}=-\mathbf{x}_{i}^{\prime 2}=-\gamma_{i 00}
$$

We start with the following action

$$
S=-\sum_{i} \mu_{i} \int d t d \sigma \sqrt{-\left|\gamma_{i}\right|} \theta\left(s_{i}(t)-\sigma\right)
$$

where $\left|\gamma_{i}\right|$ is the determinant of the world sheet metric of the $i$-th string. We are using the convention that the position of the junction on the $i$-th string is given by $s_{i}(t)$. It is assumed that $\sigma$ is increasing towards the junction. We can impose a lower cutoff on $\sigma$, which would correspond to the physical length of the string under consideration. The equation of motion for $s_{i}(t)$ and the conditions for junction formation have been studied in $[22,23,8]$.

The energy-momentum tensor for the action given by Eq. (3.4) is obtained by varying the action with respect to the background metric $g_{\mu \nu}$, with the result

$\delta_{g_{\mu \nu}} S=-\frac{1}{2} \sum_{i} \mu_{i} \int d t d \sigma \sqrt{-\left|\gamma_{i}\right|} \gamma^{a b} \partial_{a} X_{i}^{\mu} \partial_{b} X_{i}^{\nu} \theta\left(s_{i}(t)-\sigma\right) \delta g_{\mu \nu} \equiv-\frac{1}{2} \int d^{4} x T^{\mu \nu} \delta g_{\mu \nu}(3.5$

which gives

$$
\begin{aligned}
T^{\mu \nu}(x) & =\sum_{i} \mu_{i} \int d t d \sigma \sqrt{-\left|\gamma_{i}\right|} \gamma^{a b} X_{i, a}^{\mu} X_{i, b}^{\nu} \theta\left(s_{i}(t)-\sigma\right) \delta^{(4)}\left(x-X_{i}\right) \\
& =\sum_{i} \mu_{i} \int d t d \sigma\left(\dot{X}^{\mu} \dot{X}^{\nu}-X^{\prime \mu} X^{\prime \nu}\right) \theta\left(s_{i}(t)-\sigma\right) \delta^{(4)}\left(x-X_{i}\right) .
\end{aligned}
$$


Having obtained the energy-momentum tensor, we can use the standard formalism for calculating GW emission from a source [24]. The derivation in [24] is for a source which is localized in space. To justify the application of the formalism to the case of a long string, we can imagine considering first short wave-trains on the string, in which case the formalism of [24] applies as it was initially derived, and then taking the limit in which the length of the wave trains increases. This limit does not lead to any problems when applying for formalism . According to this formalism, the power emitted in direction $\mathbf{k}$ per solid angle $\Omega$, integrating over the frequencies $\omega$ of the emitted waves, is given by

$$
\frac{d E}{d \Omega}=2 G \int_{0}^{\infty} d \omega \omega^{2}\left[T^{\lambda \nu^{*}}(k) T_{\lambda \nu}(k)-\frac{1}{2}\left|T_{\lambda}^{\lambda}(k)\right|^{2}\right],
$$

where $G$ is Newton's gravitational constant and $T_{\lambda \nu}(k)$ is the Fourier transform of $T_{\lambda \nu}(t, \mathbf{x})$

$$
T_{\mu \nu}(k)=\frac{1}{2 \pi} \int d^{4} x T_{\mu \nu}(x) e^{i k . x} .
$$

In conformal temporal gauge the solution of the string equations of motion

$$
\ddot{X}^{\mu}-X^{\prime \mu}=0
$$

can be represented by the combination of left-moving and right-moving modes:

$$
X_{i}^{\mu}=\frac{1}{2}\left(a_{i}^{\mu}(v)+b_{i}^{\mu}(u)\right) \quad, \quad a_{i}^{\prime 2}=b_{i}^{\prime 2}=0 .
$$

where $v=\sigma+t$ and $u=\sigma-t$ are the light-cone coordinates. 
Since we need the components of the energy-momentum tensor in Fourier space, it is useful to replace the $\theta$ function by its Fourier representation which is

$$
\theta(x)=\frac{1}{2 \pi i} \int_{-\infty}^{\infty} \frac{d \ell e^{i \ell x}}{\ell-i \varepsilon} \quad, \quad \varepsilon \rightarrow 0^{+}
$$

Inserting this into Eq. (3.6), we find

$$
\begin{aligned}
T^{\mu \nu}(k)=\sum_{j} \frac{\mu_{i}}{8 \pi} \frac{1}{2 \pi i} \int_{-\infty}^{\infty} \frac{d \ell}{\ell-i \varepsilon} & \int d u d v\left(a_{j}^{\prime \mu} b_{j}^{\prime \nu}+a_{j}^{\prime \nu} b_{j}^{\prime \mu}\right) \\
& \times \exp \left[i \ell s_{j}(u, v)-\frac{i \ell}{2}(u+v)+\frac{i}{2} k \cdot\left(a_{j}+\left(b_{3}\right) .12\right)\right.
\end{aligned}
$$

In the first two examples in the following section, we consider cases when the junction remains stationary, corresponding to $s_{i}=0$. In this case, one obtains

$$
T^{\mu \nu}(k)=\sum_{j} \frac{\mu_{j}}{8 \pi} \frac{1}{2 \pi i} \int_{-\infty}^{\infty} \frac{d \ell}{\ell-i \varepsilon}\left(A_{j}^{\mu}(k, \ell) B_{j}^{\nu}(k, \ell)+A_{j}^{\nu}(k, \ell) B_{j}^{\mu}(k, \ell)\right)
$$

where

$$
\begin{aligned}
A_{j}^{\mu}(k, \ell) & \equiv \int_{-L / 2}^{L / 2} d v a_{j}^{\prime \mu}(v) \exp \left[i k . a_{j}(v) / 2-i \ell v / 2\right] \\
B_{j}^{\mu}(k, \ell) & \equiv \int_{-L / 2}^{L / 2} d u b_{j}^{\prime \mu}(u) \exp \left[i k . b_{j}(u) / 2-i \ell u / 2\right]
\end{aligned}
$$

where $L$ is the physical length of the string being considered (since the GW emission comes from regions where the wave trains are non-vanishing, effectively $L$ can be taken as the length on the string which corresponds to the region where the wavetrains are localized), and we assumed the mid point of the string is at world sheet coordinates $u=v=0$. 
To calculate $A_{i}^{\mu}(k, \ell)$ and $B_{i}^{\mu}(k, \ell)$ we follow the formalism of [27]. We assume that on each string there are left-moving and right-moving wave-trains of lengths $L_{i}=N_{a}^{i} \lambda_{a}^{i}$ and $L_{i}=N_{b}^{i} \lambda_{b}^{i}$ for integers $N_{a}^{i}$ and $N_{b}^{i}$, where $\lambda_{a(b)}^{i}=2 \pi / \kappa_{a(b)}^{i}$ are the wavelengths of the left(right)-moving wave-trains on each string.

We are interested in GW emission from the excitations of the strings and neglect the contributions of the straight parts of the strings to $T^{\mu \nu}$. To establish our notation, the contributions to a quantity $Q$ from the string excitations are denoted by $\delta Q$. For example, $a_{i}^{\mu} \rightarrow a_{i}^{\mu}+\delta a_{i}^{\mu}$ and so on. Discarding the contributions from the straight parts of the strings (which do not contribute to gravitational radiation), one obtains for the fluctuating part of $T^{\mu \nu}$ :

$$
\delta T^{\mu \nu}(k)=\sum_{j} \frac{\mu_{j}}{8 \pi} \frac{1}{2 \pi i} \int_{-\infty}^{\infty} \frac{d \ell}{\ell-i \varepsilon}\left(\delta A_{j}^{\mu} \delta B_{j}^{\nu}+\delta A_{j}^{\nu} \delta B_{j}^{\mu}\right),
$$

where

$$
\delta A_{j}^{\mu}(k, \ell)=\int_{-L / 2}^{L / 2} d v e^{i v \hat{K}_{+}^{j} / 2}\left(\delta a_{j}^{\prime \mu}+\frac{i}{2} a_{j}^{\prime \mu} k . \delta a_{j}\right)
$$

and $\delta B^{\mu}(k, \ell)$ is given by a similar expression. Here

$$
\hat{K}_{+}^{j} \equiv K_{+}^{j}-\ell \quad, \quad K_{+}^{j} \equiv k \cdot a_{j}^{\prime}
$$

with $\hat{K}_{-}$and $K_{-}$defined similarly for the right-movers. 
For the case where $s_{i} \neq 0$, such as in the third example in the next section, we obtain

$$
\begin{aligned}
& \delta T^{\mu \nu}(k)=\sum_{j} \frac{\mu_{j}}{8 \pi} \frac{1}{2 \pi i} \int_{-\infty}^{\infty} \frac{d \ell}{\ell-i \varepsilon}\left\{\left(\delta A_{j}^{\mu} \delta B_{j}^{\nu}+\delta A_{j}^{\nu} \delta B_{j}^{\mu}\right)\right. \\
& +\quad i \ell \int d u d v s_{j} e^{i \hat{K}_{+}^{j} v / 2} e^{i \hat{K}_{-}^{j} u / 2}\left[\left(a_{j}^{\prime \mu} \delta b_{j}^{\prime \nu}+b_{j}^{\prime \nu} \delta a_{j}^{\prime \mu}+\mu \leftrightarrow \nu\right)+\frac{i}{2} k .\left(\delta a_{j}+\delta b_{j}\right)\left(a_{j}^{\prime \mu} b_{j}^{\prime \nu}+a_{j}^{\prime \nu} b_{j}^{\prime \mu}\right)\right] \\
& \left.-\frac{\ell^{2}}{2} \int d u d v s_{j}^{2} e^{i \hat{K}_{+}^{j} v / 2} e^{i \hat{K}_{-}^{j} u / 2}\left(a_{j}^{\prime \mu} b_{j}^{\prime \nu}+a_{j}^{\prime \nu} b_{j}^{\prime \mu}\right)\right\}
\end{aligned}
$$

\subsection{Examples}

In this section we employ the formalism presented in the last section to calculate GW emission for different examples.

\subsubsection{A semi-infinite string attached to the wall}

The first example we would like to consider is gravitational radiation from a semi-infinite string attached to a rigid wall. An incoming perturbation is coming from infinity, hits the wall and gets reflected. This creates wave-trains of both leftmover and right-mover on the string. This problem is in spirit very similar to the problem of two left-moving and right-moving wave-trains propagating on an infinite string studied by Siemens and Olum [27].

The non-fluctuating string configuration is given by

$$
a^{\prime \mu}=(1, \mathbf{e}) \quad, \quad b^{\prime \mu}=(-1, \mathbf{e})
$$

where the unit vector e represents the orientation of the string. We could simply take the vector e to be along the $z$ axis. However, in order to establish a formalism which can also be applied to the next examples involving strings oriented in different 
directions, we keep the vector e unspecified. The perturbations on the string are given by

$$
\delta a^{\prime \mu}=\epsilon_{a} \mathbf{f} \cos \left(\kappa_{a} v\right) \quad, \quad \delta b^{\prime \mu}=\epsilon_{b} \mathbf{f} \cos \left(\kappa_{a} u\right)
$$

where $\epsilon_{a(b)}$ are small numbers controlling the amplitude of the perturbations, $\kappa_{a(b)}$ are the frequencies of the left(right)-moving perturbations and $\mathbf{f}$ is a unit vector indicating the polarization of the perturbations with e.f $=0$. In this example we know that $\epsilon_{a}=\epsilon_{b}$ and $\kappa_{a}=\kappa_{b}$. However, in order to keep the formalism general we have not made these identifications.

Calculating $\delta A^{\mu}$, one obtains

$$
\delta \vec{A}=\delta A^{0}\left(\mathbf{e}-\frac{\hat{K}_{+}}{\mathbf{k} . \mathbf{f}}\right) \quad, \quad \delta A^{0}=-4(-1)^{N_{a}} \epsilon_{a} \mathbf{k . f} \frac{\sin \left(L \hat{K}_{+} / 4\right)}{\hat{K}_{+}^{2}-4 \kappa_{a}^{2}}
$$

with a similar expressions for $\delta B^{\mu}$ with $\hat{K}_{+}$replaced by $\hat{K}_{-}$.

To calculate $\delta T^{\mu \nu}(k)$, we need to plug $\delta A^{\mu}(k, \ell)$ and $\delta B^{\mu}(k, \ell)$ into Eq. (3.15) and integrate over $\ell$. There are five poles at

$$
\ell_{1}=i \varepsilon \quad, \quad \ell_{2,3}=K_{+} \pm 2 \kappa_{a} \quad, \quad \ell_{4,5}=K_{-} \pm 2 \kappa_{a}
$$

One can easily check that only the residue at $\ell_{1}$ gives a non-zero contribution to $\delta T^{\mu \nu}(k)$ and the other residues vanish. For example, calculating the residue at $\ell=\ell_{2}$, one obtains that $\delta T^{\mu \nu}(k) \propto \sin \left(L \kappa_{a} / 2\right)=\sin \left(N_{a} \pi\right)=0$.

Calculating the residue at $\ell=\ell_{1}$, one obtains

$$
\delta T^{\mu \nu}(k)=\frac{\mu_{1}}{8 \pi}\left[\delta A^{\mu}(k, 0) \delta B^{\nu}(k, 0)+\delta A^{\nu}(k, 0) \delta B^{\mu}(k, 0)\right]
$$


Noting that $\delta A^{\mu}(k, 0)$ and $\delta B^{\mu}(k, 0)$ are real, one obtains

$$
\begin{aligned}
\frac{d E}{d \Omega d \omega} & =\frac{G \mu_{1}^{2}}{16 \pi^{2}} \omega^{2} \delta A(k, 0)^{2} \delta B(k, 0)^{2} \\
& =\frac{16 G \mu_{1}^{2}}{\pi^{2}} \omega^{2} \epsilon_{a}^{2} \epsilon_{b}^{2} K_{+}^{2} K_{-}^{2} \frac{\sin ^{2}\left(L K_{+} / 4\right)}{\left(K_{+}^{2}-4 \kappa_{a}^{2}\right)^{2}} \frac{\sin ^{2}\left(L K_{-} / 4\right)}{\left(K_{-}^{2}-4 \kappa_{b}^{2}\right)^{2}}
\end{aligned}
$$

One interesting result which emerges from the above is that the combination k.f drops from the numerator and denominator of the above expression. This indicates that the GW power emission is independent of the polarization of the incoming waves. This feature will also show up in next example.

To exploit the symmetry of the problem, now we assume that the string is oriented along the $z$ axis, so k.e $=\omega \cos \theta$, where $\theta$ is defined as the angle between the vector $\mathbf{k}$ and the orientation of string. On the other hand, calculating $K_{+}$and $K_{-}$, we get

$$
K_{+}=\omega(1-\cos \theta) \quad, \quad K_{-}=-\omega(1+\cos \theta) .
$$

As in [27], changing the coordinates from $(\omega, \cos \theta)$ to $\left(K_{+}, K_{-}\right)$, and noting that $2 \omega=K_{+}-K_{-}$and $d \omega d(\cos \theta)=d K_{+} d K_{-} / 2 \omega$, one obtains

$$
\frac{d E}{d \phi}=\frac{4 G \mu_{1}^{2}}{\pi^{2}} \epsilon_{a}^{2} \epsilon_{b}^{2} \int d K_{+} d K_{-}\left(K_{+}-K_{-}\right) K_{+}^{2} K_{-}^{2} \frac{\sin ^{2}\left(L K_{+} / 4\right)}{\left(K_{+}^{2}-4 \kappa_{a}^{2}\right)^{2}} \frac{\sin ^{2}\left(L K_{-} / 4\right)}{\left(K_{-}^{2}-4 \kappa_{b}^{2}\right)^{2}}
$$

Here $\phi$ is defined as the azimuthal angle around the string.

The above integral can be performed using the following approximations for large $N_{a}$ [27]

$$
\frac{\sin ^{2}\left(N_{a} x^{a} / 2\right)}{\sin ^{2}\left(x^{a} / 2\right)}=2 \kappa_{a} N_{a} \sum_{n=-\infty}^{\infty} \delta\left(K_{+}-2 n \kappa_{a}\right)
$$


where $x_{a} \equiv \lambda_{a} K_{+} / 2$. A similar identity is also holds for right-movers with $K_{+} \rightarrow$ $K_{-}, N_{a} \rightarrow N_{b}$ and $n \rightarrow m$. Using this identity, Eq. (3.26) yields

$$
\frac{d E}{d \phi}=\frac{2 G \mu_{1}^{2}}{\pi^{2}} \frac{N_{a} N_{b}}{\kappa_{a} \kappa_{b}} \sum_{m, n} \frac{n^{2} m^{2}\left(n \kappa_{a}-m \kappa_{b}\right) \sin ^{2}(n \pi) \sin ^{2}(m \pi)}{\left(n^{2}-1\right)^{2}\left(m^{2}-1\right)^{2}} .
$$

Knowing that $K_{+} \geq 0, K_{-} \leq 0$, one can see that only $n=-m=1$ contribute in the summation in Eq. (3.28) and one obtains

$$
\frac{d E}{d \phi}=\frac{G \mu_{1}^{2}}{8} N_{a} N_{b} \pi^{2} \epsilon_{a}^{2} \epsilon_{b}^{2} \frac{\kappa_{a}+\kappa_{b}}{\kappa_{a} \kappa_{b}}
$$

We are interested in the power radiated per unit of length, $d P / d l$, which is obtained by dividing the above expression by the world-sheet volume of the string, $L_{a} L_{b} / 2$. After integrating over the angle $\phi$ and noting that $\epsilon_{a}=\epsilon_{b}$ and $\kappa_{a}=\kappa_{b}$, we obtain

$$
\frac{d P}{d l}=\frac{G \mu_{1}^{2} \pi}{16} \epsilon_{a}^{4} \kappa_{a}
$$

Note that our result is identical to the power radiated per unit of length obtained in [27] for an infinite string. This is not surprising since our semi-infinite string locally looks identical to an infinite string. The only difference is at the junction with the wall - but since that point is not moving it does not contribute to the power of gravitational radiation. Thus, we expect that our result for a semi-infinite string agrees with that of [27] for an infinite string (there is a factor of $1 / 2$ mistake in the original version of Eq. (72) of [27] which is corrected in the printed version. Taking that into account, our result here agrees with their Eq. (36)). 
As in [27] the power radiation is dominated by lower harmonics. Also for $n=$ $-m=1$ we note that $\omega=2 \kappa_{a}$, so the frequency of the radiation is twice of the frequency of the incoming wave.

\subsubsection{Strings at a junction}

In this section we consider the problem of GW emission from strings at a junction. Three semi infinite strings form a stationary junction. There is an incoming right moving excitation on one string, say String 1. After the wave hits the junction, part of it is transferred to Strings 2 and 3, while part of the incoming wave is reflected along String 1 (see [23] for the details of the dynamics). Depending on the polarization of the incoming wave, the junction may stay stationary, corresponding to $\delta s_{i}(t)=0$, or the junction may dislocate along the strings corresponding to $\delta s_{i}(t) \neq 0$.

To be specific, suppose that the strings are in the $x-y$ plane and their orienta-

tions are given by $\mathbf{e}_{i}=\left(\cos \theta_{i}, \sin \theta_{i}, 0\right)$ where $\theta_{i}$ is the angle of the $i$-th string with the $x$-axis. This gives

$$
\begin{aligned}
a_{i}^{\prime \mu} & \equiv\left(1, \mathbf{a}_{i}\right)=\left(1, \mathbf{e}_{i}\right) \\
b_{i}^{\prime \mu} & \equiv\left(-1, \mathbf{b}_{i}\right)=\left(-1, \mathbf{e}_{i}\right),
\end{aligned}
$$

where $\mathbf{a}_{i}\left(\mathbf{b}_{i}\right)$ indicates the spatial part of $a_{i}^{\mu}\left(b_{i}^{\mu}\right)$. The relations $\sum \mu_{i} \cos \theta_{i}=\sum \mu_{i} \sin \theta_{i}=$ 0 also must be satisfied if the junction is to be stationary (this is due to the force balance condition). 
Now suppose there is a small incoming excitation on one string, say String 1, with

$$
\delta \mathbf{b}_{1}^{\prime}(u)=\epsilon \mathbf{f}_{1} \cos (\kappa u)
$$

and $\delta \mathbf{b}_{2}^{\prime}=\delta \mathbf{b}_{3}^{\prime}=0$. Here $\epsilon \ll 1$ is a dimensionless parameter controlling the amplitude of the perturbation.

\subsubsection{The case $s_{i}(t)=0$}

The simplest case is when $\mathbf{f}_{1}=(0,0,1)$. This has the advantage that the junction does not dislocate on the strings: $\delta \dot{s}_{i}=0$ [23] and we can use the formalism developed in the previous section. Following [23], one finds

$$
\delta \mathbf{a}_{1}^{\prime}=\frac{\nu_{1}}{\mu} \epsilon \mathbf{f}_{1} \cos (\kappa v) \quad, \quad \delta \mathbf{a}_{2}^{\prime}=\delta \mathbf{a}_{3}^{\prime}=\frac{2 \mu_{1}}{\mu} \epsilon \mathbf{f}_{1} \cos (\kappa v)
$$

where

$$
\mu \equiv \mu_{1}+\mu_{2}+\mu_{3} \quad, \quad \nu_{1} \equiv \mu_{2}+\mu_{3}-\mu_{1}
$$

With this initial condition, one sees that only $\delta B_{1}^{\mu}$ is non-zero. Furthermore, $\delta T^{\mu \nu}(k)$ is as given in Eq. (3.23), with $\delta A_{1}^{\mu}$ given as in Eq.(3.21). Thus, $d E / d \Omega d \omega$ has the same form as Eq. (3.24). We note that String 1 is in $x-y$ plane. But we can label the coordinate (or perform a coordinate transformation) such that String 1 is along the $z$-direction. To calculate the power radiated, we can simply use Eq. (3.29) with the identification $\epsilon_{b}=\epsilon$ and $\epsilon_{a}=\epsilon \nu_{1} / \mu$, and as before, we note that $\phi$ is defined as the azimuthal angle around String 1 . The power radiated per unit of 
length, using Eq. (3.29), therefore is

$$
\frac{d P}{d l}=\frac{G \mu_{1}^{2} \pi}{16} \frac{\nu_{1}^{2}}{\mu^{2}} \epsilon^{4} \kappa
$$

One may wonder why this result has the same form as that in the previous example where a semi-infinite strings was attached to a rigid wall. The reason is that here the junction plays the role of the rigid wall. Indeed, the fact that the junction remains stationary makes this analogy more manifest. The effect of Strings 2 and 3 is to let parts of incoming waves be transferred to them. This has the effect that $\epsilon_{a} \neq \epsilon_{b}$.

\subsubsection{The case $s_{i}(t) \neq 0$}

Now we consider the case when the polarization of the incoming perturbation is in the $x y$-plane. Then $s_{i}$ will also oscillate and the junction does not stay at a fixed position on each string [23]. As in [23] we assume

$$
\mathbf{f}_{i}=\left(-\sin \theta_{i}, \cos \theta_{i}, 0\right)
$$

and $\mathbf{e}_{i} \cdot \mathbf{f}_{i}=0$ for each string. As before, the incoming perturbation is along String 1 and is given by

$$
\delta \mathbf{b}_{1}^{\prime}(u)=\epsilon \mathbf{f}_{1} \cos (\kappa u) .
$$

Following [23], one obtains

$$
\delta \mathbf{a}_{1}^{\prime}=-\frac{\nu_{1}}{\mu} \epsilon \mathbf{f}_{1} \cos (\kappa v) \quad, \quad \delta \mathbf{a}_{2,3}^{\prime}=\frac{2 \mu_{1}}{\mu} \epsilon \mathbf{f}_{2,3} \cos (\kappa v),
$$


and

$$
\delta s_{1}=\frac{\left(\mu_{2}-\mu_{3}\right) \nu_{1}}{\kappa \Delta} \epsilon \sin (\kappa t) \quad, \quad \delta s_{2}=-\delta s_{3}=-\frac{\mu_{1} \nu_{1}}{\kappa \Delta} \epsilon \sin (\kappa t)
$$

with $\nu_{1}$ as given in Eq. (3.34) and $\Delta=\sqrt{\mu \nu_{1} \nu_{2} \nu_{3}}$, where $\nu_{2,3}$ are defined like $\nu_{1}$ with the appropriate permutations.

With these forms of $\delta s_{i}$, one can show (see the Appendix) that both integrals containing the linear and the quadratic powers of $s_{i}(t)$ in $\delta T_{\mu \nu}(k)$ in Eq. (3.18) vanish.

One sees that $\delta T^{\mu \nu}$ is of the same form as Eq. (3.23) with $\delta A_{1}^{\mu}$ given as in Eq.(3.21) . Like in previous example, the effect of the junction is to make $\epsilon_{a} \neq \epsilon_{b}$. On the other hand, since the amplitude of $\delta a_{1}^{\mu}$ is the same as in the previous example where the polarization was along the $z$ axis, we find that the power radiated is the same as before, given by Eq. (3.35).

The fact that the power of gravitational radiation when the polarization $\mathbf{f}_{1}$ is coplanar with the strings is the same as when the polarization is perpendicular to the plane of the strings may seem surprising. However, as mentioned in [23], one can consider the excitations on the strings as the propagation of massless particles. Using conservation of energy, one can check that the transmission and reflection indices for both polarizations are the same.

\subsection{Discussion}

In this paper, gravitational wave (GW) emission from strings at a stationary junction has been studied. We considered the simple case when three co-planar semi-infinite strings form a stationary junction. A purely left-moving wave, excited 
on one string, travels towards the junction. Part of it is reflected from the junction while the rest is transferred to other strings. The role of the junction therefore is to mix the left-moving and right-moving excitations which are necessary for GW emission.

We found that power of gravitational radiation is independent of the polarization of the incoming wave. Furthermore, its magnitude is proportional to the frequency of the incoming wave. This means that excitations of higher frequencies (shorter wavelengths) die out faster than excitations with lower frequencies (longer wavelengths).

In $[27,25,26]$ the gravitational back-reaction effects on the small scale structure present on a long string are studied. Here we shall briefly apply their formalism to our case. An excitation of the form Eq. (3.32) leads to a change $\delta \mu$ in the mass per unit length of the string. This change takes the form

$$
\delta \mu \sim \mu_{1} \epsilon^{2}
$$

The energy loss via gravitational radiation given by Eq. (3.30) or Eq. (3.35) leads to a decrease of this contribution:

$$
\frac{d}{d t}(\delta \mu)=-\frac{d P}{d z}=-\frac{G \mu_{1}}{8 \lambda} \pi^{2} \frac{\nu_{1}^{2}}{\mu^{2}} \epsilon^{2} \delta \mu .
$$

This differential equation has the solution

$$
\delta \mu \sim \exp (-t / \tau)
$$


where

$$
\tau=\frac{8 \lambda}{\pi^{2} G \mu_{1}} \frac{\mu^{2}}{\epsilon^{2} \nu_{1}^{2}}
$$

Excitations which survive until the present time $t_{0}$ are characterized by $\tau>t_{0}$. Taking $\epsilon \lesssim 1$, the minimum wavelength of excitations that can survive is thus approximately given by

$$
\lambda_{\min } \sim G \mu_{1} \frac{\nu_{1}^{2}}{\mu^{2}} t_{0}
$$

while on smaller scales the wiggles are exponentially suppressed.

In this analysis we have considered monochromatic wave in the form of Eq. (3.32). In [25] (see also [26]) the estimation of back-reaction was generalized to the case when higher harmonics of the initial Fourier modes on the long string are present and when not all the modes interact with all of the other modes. In this case, it was shown that the minimum wavelength is given by

$$
\lambda_{\min }=\left(G \mu_{1}\right)^{n} t_{0}
$$

where $n=3 / 2,5 / 2$ for radiation and matter dominated eras, respectively.

In this work we have considered GW emission from three co-planar strings forming a junction and assuming that excitations are originally generated on one string leg. It would be interesting to generalize this exercise to more realistic cases of an arbitrary number of strings in a junction when incoming waves of arbitrary frequencies and amplitudes are excited on each string. It would be interesting to see if the 
results of [27] hold, where it was shown that GW emission from left-moving and rightmoving wave-trains on an infinite string is zero if the wave-trains have significantly different frequencies and amplitudes.

\subsection{Acknowledgments}

We would like to thank E. Copeland, T. Kibble, X. Siemens and D. Steer for useful comments.

\subsection{Appendix}

In this Appendix we show that the integrals containing the powers of $s_{i}(t)$ in $\delta T_{\mu \nu}(k)$ in Eq. (3.18) vanish. To see this, consider the term quadratic in $s_{i}^{2}$ when the corresponding integral is

$$
\begin{aligned}
& \int_{-\infty}^{\infty} \frac{\ell^{2} d \ell}{\ell-i \varepsilon} \int d u d v s_{i}^{2} e^{i \hat{K}_{+} v / 2} e^{i \hat{K}_{-} u / 2} \\
& \sim \int_{-\infty}^{\infty} \frac{\ell^{2} d \ell}{\ell-i \varepsilon} \int d u d v e^{i \hat{K}_{+} v / 2} e^{i \hat{K}_{-} u / 2} \sin ^{2}\left(\frac{\kappa}{2}(v-u)\right) .
\end{aligned}
$$

The integral further simplifies to

$$
\int_{-\infty}^{\infty} \frac{\ell^{2} d \ell}{\ell-i \varepsilon} \int d u d v e^{i \hat{K}_{+} v / 2} e^{i \hat{K}_{-} u / 2}(1-\cos \kappa u \cos \kappa v-\sin \kappa u \sin \kappa v)
$$

The first term in the above bracket gives

$$
\begin{aligned}
& \int_{-\infty}^{\infty} \frac{\ell^{2} d \ell}{\ell-i \varepsilon} \int d u d v e^{i \hat{K}_{+} v / 2} e^{i \hat{K}_{-} u / 2} \\
& \sim \int_{-\infty}^{\infty} \frac{\ell^{2} d \ell}{\ell-i \varepsilon} \frac{\sin \left(\hat{K}_{+} L / 4\right)}{\hat{K}_{+}} \frac{\sin \left(\hat{K}_{-} L / 4\right)}{\hat{K}_{-}} .
\end{aligned}
$$


This integral has three poles at $\ell=i \varepsilon, \hat{K}_{+}=0$ and $\hat{K}_{-}=0$. However, the residues at all three poles vanish. Performing the integrals for the other two terms in the bracket in Eq. (3.47), one can check that the residues at the poles vanish.

In conclusion, the integral in Eq. (3.47) and correspondingly the integral in Eq. (3.18) containing terms of second power in $s_{i}(t)$ vanish. Following the same strategy, one can check that the integral in Eq. (3.18) containing terms of linear power in $s_{i}(t)$ also vanishes. 


\section{REFERENCES}

[1] S. H. Henry Tye, "Brane inflation: String theory viewed from the cosmos," Lect. Notes Phys. 737, 949 (2008) [arXiv:hep-th/0610221].

[2] T. W. B. Kibble, "Cosmic strings reborn?," astro-ph/0410073.

[3] A. C. Davis and T. W. B. Kibble, "Fundamental cosmic strings," Contemp. Phys. 46, 313 (2005) [arXiv:hep-th/0505050].

[4] M. Sakellariadou, "Cosmic Superstrings," arXiv:0802.3379 [hep-th].

[5] B. Shlaer and M. Wyman, "Cosmic superstring gravitational lensing phenomena: Predictions for networks of (p,q) strings," Phys. Rev. D 72, 123504 (2005) [arXiv:hep-th/0509177].

[6] E. J. Copeland, T. W. B. Kibble and D. A. Steer, "Collisions of strings with Y junctions," Phys. Rev. Lett. 97, 021602 (2006) [arXiv:hep-th/0601153].

[7] E. J. Copeland, T. W. B. Kibble and D. A. Steer, "Constraints on string networks with junctions," Phys. Rev. D 75, 065024 (2007) [arXiv:hep-th/0611243].

[8] E. J. Copeland, H. Firouzjahi, T. W. B. Kibble and D. A. Steer, "On the Collision of Cosmic Superstrings," Phys. Rev. D 77, 063521 (2008) [arXiv:0712.0808 [hepth]].

[9] R. Brandenberger, H. Firouzjahi and J. Karouby, Phys. Rev. D 77, 083502 (2008) [arXiv:0710.1636 [hep-th]].

[10] A. Avgoustidis and E. P. S. Shellard, "Velocity-Dependent Models for NonAbelian/Entangled String Networks," arXiv:0705.3395 [astro-ph].

[11] A. Rajantie, M. Sakellariadou and H. Stoica, "Numerical experiments with p Fand q D-strings: the formation of (p,q) bound states," JCAP 0711, 021 (2007) [arXiv:0706.3662 [hep-th]]. 
[12] J. Urrestilla and A. Vilenkin, "Evolution of cosmic superstring networks: a numerical simulation," JHEP 0802, 037 (2008) [arXiv:0712.1146 [hep-th]].

[13] A. C. Davis, W. Nelson, S. Rajamanoharan and M. Sakellariadou, "Cusps on cosmic superstrings with junctions," arXiv:0809.2263 [hep-th].

[14] L. Leblond and M. Wyman, "Cosmic Necklaces from String Theory," Phys. Rev. D 75, 123522 (2007) [arXiv:astro-ph/0701427].

[15] K. Dasgupta, H. Firouzjahi and R. Gwyn, "Lumps in the throat," JHEP 0704, 093 (2007) [arXiv:hep-th/0702193].

[16] T. Suyama, "Exact gravitational lensing by cosmic strings with junctions," Phys. Rev. D 78, 043532 (2008) [arXiv:0807.4355 [astro-ph]].

[17] M. B. Hindmarsh and T. W. B. Kibble, "Cosmic strings," Rept. Prog. Phys. 58, 477 (1995) [arXiv:hep-ph/9411342].

[18] "Cosmic Strings and Other Topological Defects, A. Vilenkin and E. P. S. Shellard, Cambridge University Press, 1994.

[19] T. Damour and A. Vilenkin, "Gravitational radiation from cosmic (super)strings: Bursts, stochastic background, and observational windows," Phys. Rev. D 71, 063510 (2005) [arXiv:hep-th/0410222].

[20] X. Siemens, V. Mandic and J. Creighton, "Gravitational wave stochastic background from cosmic (super)strings," Phys. Rev. Lett. 98, 111101 (2007) [arXiv:astro-ph/0610920];

X. Siemens, J. Creighton, I. Maor, S. Ray Majumder, K. Cannon and J. Read, "Gravitational wave bursts from cosmic (super)strings: Quantitative analysis and constraints," Phys. Rev. D 73, 105001 (2006) [arXiv:gr-qc/0603115].

[21] M. Sakellariadou, "Gravitational waves emitted from infinite strings," Phys. Rev. D 42, 354 (1990) [Erratum-ibid. D 43, 4150 (1991)].

[22] M. Hindmarsh, "Gravitational radiation from kinky infinite strings," Phys. Lett. B 251, 28 (1990).

[23] X. Siemens and K. D. Olum, "Gravitational radiation and the small-scale structure of cosmic strings," Nucl. Phys. B 611, 125 (2001) [Erratum-ibid. B 645, 367 (2002)] [arXiv:gr-qc/0104085]. 
[24] S. Weinberg, 'Gravitation and cosmology: principles and applications of the general theory of relativity", Wiley, New York, 1972.

[25] X. Siemens, K. D. Olum and A. Vilenkin, "On the size of the smallest scales in cosmic string networks," Phys. Rev. D 66, 043501 (2002) [arXiv:gr-qc/0203006].

[26] J. Polchinski and J. V. Rocha, "Cosmic string structure at the gravitational radiation scale," Phys. Rev. D 75, 123503 (2007) [arXiv:gr-qc/0702055]. 


\section{CHAPTER 4 Conclusion and prospects}

There are different ways of detecting cosmic strings if they exist. We've studied, the lensing,the KS effect and the gravitational radiation in the case of junction. This could allow a possible detection by future telescope and be in favour of the existence of string theory since junctions mainly exist in network of cosmic superstrings. The deformation of space-time due to a junction is very similar to the one straight cosmic string junction. Using a superposition principle we have to add up the contribution due to each piece of strings. In chapter 2, we computed the metric and found that the spacetime around a junction is, like in the single string case, flat. We then study the lensing due to a junction and saw that their would be multiple images of the same object close to a cosmic string junction. More precisely,we have computed the angle of deviation for light rays passing through a junction: It depends on the tension of each of the strings forming the junction and of the relative angle between each other. We then computed the shift in the frequency passing through a junction and deduced from it the anisotropy of the background temperature, called Kaiser-Stebbins effect. In the last part we find the gravitationnal power that would be emitted by a single string attached to a wall on which there is an incomming perturbation coming from infinity and we generalise this result to the coplanar and stationary junctions case. This case is interesting since at the junction point, a part of the wave will be reflected (as it is by the wall in the semi-string case). This is what create the emission of 
gravitationnal wave : we need a coupling between left- and right- movers on the strings. We found that the power depends on the frequency of the wave and its amplitude. The power per unit length is identical to the one emitted by infinite strings in [27] All these effects, are detectable and if we observe them, it would be a progress and a clue that we are on the right track with string theory.

Another aspect of cosmic strings is the study of a network. In this case, simulations have to take into account collisions, intercommutation, loop creation and radiation in order to correctly predict the evolution of a string network. Collision of straight strings have already been studied in $[22,23]$. An interesting process to study, is the collision of loop of strings. In this case recent simulations seems to show a possible unzipping of the junction. For straight strings, it is shown in the two papers cited above, that the third string linking the two initial colliding strings just grow without ever getting smaller. We are going to generalize their work to loops of strings and see if, contrary to the case of straight strings junctions, the length of the junction can decrease to zero, leading to an unzipping of the loops. A recent paper ([24]) studies possible loop configuration using Nambu-Goto action and field theory simulation. Looking more carefully to collisions could explain some of their results and better understand the evolution of a string network.

Besides the references for each chapter, the following references have been used for the introduction and the conclusion. 


\section{REFERENCES}

[1] R. H. Brandenberger and N. Turok, Phys. Rev. D 33, 2182 (1986).

H. Sato, "Galaxy Formation by Cosmic Strings," Prog. Theor. Phys. 75,1342 (1986)

A. Stebbins, "Cosmic strings and cold matter, "Astrophysical Journal, vol. 303, April 15, 1986, p. L21-L25.

[2] P. D. Mauskopf et al. [Boomerang Collaboration], Astrophys. J. 536, L59 (2000) [arXiv:astro-ph/9911444].

[3] M. Wyman, L. Pogosian and I. Wasserman, "Bounds on cosmic strings from WMAP and SDSS," Phys. Rev. D 72, 023513 (2005) [Erratum-ibid. D 73, 089905 (2006)] [arXiv:astro-ph/0503364];

[4] R. Jeannerot, "A Supersymmetric SO(10) Model with Inflation and Cosmic Strings," Phys. Rev. D 53, 5426 (1996) [arXiv:hep-ph/9509365].

[5] G. R. Dvali and S. H. H. Tye, Phys. Lett. B 450, 72 (1999) [arXiv:hep$\mathrm{ph} / 9812483]$.

C. P. Burgess, J. M. Cline, H. Stoica and F. Quevedo, "Inflation in realistic Dbrane models," JHEP 0409, 033 (2004) hep-th/0403119.

[6] S. H. Henry Tye, "Brane inflation: String theory viewed from the cosmos," Lect. Notes Phys. 737, 949 (2008) [arXiv:hep-th/0610221].

[7] E. Witten, Phys. Lett. B 153, 243 (1985).

[8] E. J. Copeland, R. C. Myers and J. Polchinski, "Cosmic F- and D-strings," JHEP 0406, 013 (2004), hep-th/0312067 ; 
[9] N. Kaiser and A. Stebbins, "Microwave Anisotropy Due To Cosmic Strings," Nature 310, 391 (1984).

[10] T. W. B. Kibble, "Cosmic strings reborn?," astro-ph/0410073.

[11] J. Polchinski, "Introduction to cosmic F- and D-strings," arXiv:hep-th/0412244.

[12] J. Polchinski, "Cosmic String Loops and Gravitational Radiation," arXiv:0707.0888 [astro-ph].

[13] "Cosmic Strings and Other Topological Defects," A. Vilenkin and E. P. S. Shellard, Cambridge University Press, 1994.

[14] G. S. Guralnik, C. R. Hagen and T. W. B. Kibble, Phys. Rev. Lett. 13, 585 (1964).

[15] A. C. Davis and T. W. B. Kibble, "Fundamental cosmic strings," Contemp. Phys. 46, 313 (2005) [arXiv:hep-th/0505050].

[16] S. H. Henry Tye, "Brane inflation: String theory viewed from the cosmos," Lect. Notes Phys. 737, 949 (2008) [arXiv:hep-th/0610221].

[17] R. H. Brandenberger, "Topological defects and structure formation," Int. J. Mod. Phys. A 9, 2117 (1994) [arXiv:astro-ph/9310041].

[18] M. B. Hindmarsh and T. W. B. Kibble, Rept. Prog. Phys. 58, 477 (1995) [arXiv:hep-ph/9411342].

[19] Don Monroe "Cosmology in the Lab," Physical Review Focus, 19 May 2006

[20] Image from Oleg Lavrentovich, Kent State University.

[21] G. Dvali and A. Vilenkin, "Formation and evolution of cosmic D-strings," JCAP 0403, 010 (2004)

[22] E. J. Copeland, T. W. B. Kibble and D. A. Steer, "Collisions of strings with Y junctions," Phys. Rev. Lett. 97, 021602 (2006) [arXiv:hep-th/0601153].

[23] E. J. Copeland, T. W. B. Kibble and D. A. Steer, "Constraints on string networks with junctions," Phys. Rev. D 75, 065024 (2007) [arXiv:hep-th/0611243].

[24] N. Bevis, E. J. Copeland, P. Y. Martin, G. Niz, A. Pourtsidou, P. M. Saffin and D. A. Steer, arXiv:0904.2127 [hep-th]. 
[25] "Cosmic strings and other defects" www.damtp.cam.ac.uk/user/gr/public/cs_top.html

[26] Smoot group cosmology "Search For Cosmic Strings ," http ://aether.lbl.gov/eunhwa_webpage_2/coverpage.html

[27] X. Siemens and K. D. Olum, "Gravitational radiation and the small-scale structure of cosmic strings," Nucl. Phys. B 611, 125 (2001) [Erratum-ibid. B 645, 367 (2002)] [arXiv:gr-qc/0104085]. 